\title{
De Novo Asymmetric Synthesis of Phoracantholide J
}

Kenneth F. Avocetien, ${ }^{\dagger, \S}$ Jiazhen J. Li, ${ }^{\dagger, \S}$ Xiaofan Liu, ${ }^{\dagger, \S}$ Yanping Wang, ${ }^{\dagger} * *$ Yalan Xing ${ }^{\perp, *}$ and George A. O'Doherty ${ }^{\dagger} * *$

${ }^{\dagger}$ Department of Chemistry and Chemical Biology, Northeastern University, Boston, Massachusetts 02115, United States

${ }^{\perp}$ Department of Chemistry, William Paterson University, 300 Pompton Rd, Wayne, New Jersey 07470, United States

Corresponding author e-mail:

* G.A.O.: email, G.ODoherty@neu.edu

*Y.W.: email, wang.yanp@husky.neu.edu

* Y.X.: email, XINGY@wpunj.edu

Supporting Information

Section A: General Information

Section B: Experimental Procedures

Section C: ${ }^{1} \mathrm{H}$ and ${ }^{13} \mathrm{C}$ NMR spectra 


\section{Section A: General Information}

${ }^{1} \mathrm{H}$ and ${ }^{13} \mathrm{C}$ NMR spectra were recorded on a Varian 400,500 or $600 \mathrm{MHz}$ spectrometer. Chemical shifts were reported relative to $\mathrm{CDCl}_{3}(\delta 7.26 \mathrm{ppm})$ for ${ }^{1} \mathrm{H}$ NMR and $\mathrm{CDCl}_{3}(\delta 77.2$ ppm) for ${ }^{13} \mathrm{C}$ NMR. Infrared (IR) spectra were obtained on a FT-IR spectrometer. Optical rotations were measured with a digital polarimeter in the solvent specified. Flash column chromatography was performed on $60-200$ or 230-400 mesh silica gel. Analytical thin-layer chromatography was performed with precoated glass-backed plates and visualized by quenching of fluorescence and by charring after treatment with $p$-anisaldehyde or potassium permanganate stain. $R_{f}$ values were obtained by elution in the stated solvent ratios. Tetrahydrofuran (THF), methylene dichloride $\left(\mathrm{CH}_{2} \mathrm{Cl}_{2}\right)$ and dimethylformamide (DMF) were dried by passing through activated alumina column with argon gas pressure. Commercial reagents were used without purification unless otherwise noted. Air- and/or moisture-sensitive reactions were carried out under an atmosphere of argon/nitrogen using oven- or flame-dried glassware and standard syringe/septa techniques. 


\section{Section B: Experimental Procedures}

\section{Hept-3-yn-2-ol, (+/-)-17}

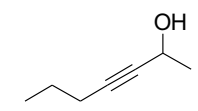

To a stirred solution of 1-pentyne $(1.50 \mathrm{~g}, 22.1 \mathrm{mmol})$ in dry THF $(44 \mathrm{~mL})$ at $-78{ }^{\circ} \mathrm{C}$ under argon was added a solution of $n$-BuLi in hexane $(11.1 \mathrm{~mL}, 26.5 \mathrm{mmol})$ dropwise. After $30 \mathrm{~min}$, acetaldehyde $(1.20 \mathrm{~mL}, 22.1 \mathrm{mmol})$ was added. The reaction was stirred for $10 \mathrm{~min}$ at $0{ }^{\circ} \mathrm{C}$ and then the resulting solution was warmed to room temperature for $50 \mathrm{~min}$. Additional $\mathrm{CH}_{2} \mathrm{Cl}_{2}$ (10 $\mathrm{mL}$ ) was added to the reaction mixture followed by saturated aqueous $\mathrm{NH}_{4} \mathrm{Cl}(20 \mathrm{~mL})$. The aqueous phase was separated and extracted with $\mathrm{CH}_{2} \mathrm{Cl}_{2}$. The combined organic layers were washed with brine, dried $\left(\mathrm{Na}_{2} \mathrm{SO}_{4}\right)$, filtered and concentrated. Chromatography on silica gel gave hept-3-yn-2-ol (+/-)-17 (2.23 g, $19.9 \mathrm{mmol}, 90 \%)$ as a colorless oil: $R_{f}=0.28(10 \%$ EtOAc/hexane); IR (neat): 3340, 2958, 2869, 2244, 1458, 1291, 1155, 1074, $893 \mathrm{~cm}^{-1} ;{ }^{1} \mathrm{H}$ NMR (600 MHz, $\left.\mathrm{CDCl}_{3}\right) \delta 4.45(\mathrm{qt}, J=6.6,1.8 \mathrm{~Hz}, 1 \mathrm{H}), 2.10(\mathrm{td}, J=6.6,1.8 \mathrm{~Hz}, 2 \mathrm{H}), 1.45$ (qt, $J=7.2,6.6 \mathrm{~Hz}, 2 \mathrm{H}), 1.35(\mathrm{~d}, J=6.6 \mathrm{~Hz}, 3 \mathrm{H}), 0.91(\mathrm{t}, J=7.2 \mathrm{~Hz}, 3 \mathrm{H}) \mathrm{ppm} ;{ }^{13} \mathrm{C} \mathrm{NMR}(150$ $\left.\mathrm{MHz}, \mathrm{CDCl}_{3}\right) \delta 84.1,82.4,58.2,24.5,21.9,20.5,13.2$ ppm. $^{1,2}$ 


\section{Hept-3-yn-2-one, 5}

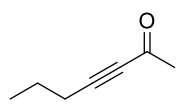

To a solution of $(+/-)-\mathbf{1 3}(4.00 \mathrm{~g}, 35.7 \mathrm{mmol})$ in THF $(100 \mathrm{~mL})$ at room temperature was added activated $\mathrm{MnO}_{2}(20.0 \mathrm{~g}, 0.23 \mathrm{~mol})$. The reaction mixture was stirred for $24 \mathrm{~h}$ then filtered through Celite and concentrated under pressure to give hept-3-yn-2-one (5) $3.53 \mathrm{~g}, 32.12 \mathrm{mmol}$, 90\%) as yellow oil. $R_{f}=0.40$ (10\% EtOAc/hexane); IR (neat): 2960, 2871, 2210, 1675, 1358, $1226 \mathrm{~cm}^{-1} ;{ }^{1} \mathrm{H}$ NMR $\left(600 \mathrm{MHz}, \mathrm{CDCl}_{3}\right) \delta 2.30(\mathrm{t}, J=7.2 \mathrm{~Hz}, 2 \mathrm{H}), 2.28(\mathrm{~s}, 3 \mathrm{H}), 1.57$ (qt, $J=$ 7.2, 7.2 Hz, 2H), $0.98(\mathrm{t}, J=7.2 \mathrm{~Hz}, 3 \mathrm{H}) \mathrm{ppm} ;{ }^{13} \mathrm{C} \mathrm{NMR}\left(150 \mathrm{MHz}, \mathrm{CDCl}_{3}\right) \delta 184.8,93.8$, 81.4, 32.6, 21.1, 20.7, 13.3 ppm. $^{2}$

(S)-Hept-3-yn-2-ol, $(S)-17$

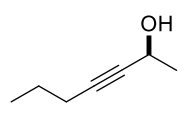

To a flask was added ketone 5 (10.8 g, $86.9 \mathrm{mmol}), 2 \mathrm{M}$ sodium formate in water $(400 \mathrm{~mL})$ and cetyl trimethylammonium bromide $(3.17 \mathrm{~g}, 8.69 \mathrm{mmol})$. The mixture was stirred at room temperature for $5 \mathrm{~min}$, and then $(S, S)$-Noyori catalyst $(105 \mathrm{mg}, 0.174 \mathrm{mmol})$ was added. The reaction mixture was allowed to be stirred for $24 \mathrm{~h}$. The reaction was diluted with water and extracted with $\mathrm{Et}_{2} \mathrm{O}$, dried $\left(\mathrm{Na}_{2} \mathrm{SO}_{4}\right)$ and concentrated under reduced pressure. The crude product was purified using silica gel flash chromatography to give alcohol $(S)-17 \quad(9.30 \mathrm{~g}$, $83.05 \mathrm{mmol}, 95 \%)$ as a colorless oil: $R_{f}=0.28(10 \%$ EtOAc/hexane $) ;[\alpha]_{\mathrm{D}}{ }^{25}=-10.3(c=0.29$, $\mathrm{MeOH}$ ); IR (neat): 3340, 2958, 2869, 2244, 1458, 1291, 1155, 1074, $893 \mathrm{~cm}^{-1} ;{ }^{1} \mathrm{H}$ NMR (600 $\left.\mathrm{MHz}, \mathrm{CDCl}_{3}\right) \delta 4.45(\mathrm{qt}, J=6.6,1.8 \mathrm{~Hz}, 1 \mathrm{H}), 2.10(\mathrm{td}, J=6.6,1.8 \mathrm{~Hz}, 2 \mathrm{H}), 1.45(\mathrm{qt}, J=7.2$, $6.6 \mathrm{~Hz}, 2 \mathrm{H}), 1.35(\mathrm{~d}, J=6.6 \mathrm{~Hz}, 3 \mathrm{H}), 0.91(\mathrm{t}, J=7.2 \mathrm{~Hz}, 3 \mathrm{H}) \mathrm{ppm} ;{ }^{13} \mathrm{C} \mathrm{NMR}(150 \mathrm{MHz}$, $\left.\mathrm{CDCl}_{3}\right) \delta 84.1,82.4,58.2,24.5,21.9,20.5,13.2 \mathrm{ppm}^{2}$

\footnotetext{
2 Trost, B. M.; Bartlett, M. J. Org. Lett. 2012, 14, 1322-1325.
} 


\section{(S)-Hept-6-yn-2-ol, 12}

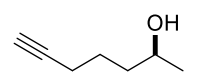

A $30 \%$ suspension of $\mathrm{KH}$ in mineral oil $(5.62 \mathrm{~g}, 140 \mathrm{mmol})$ was washed three times under argon with ether, and then the excess solvent was removed under vacuum. Dry 1,3diaminopropane $(28 \mathrm{~mL})$ was added at $0{ }^{\circ} \mathrm{C}$. The reaction was warmed slowly to room temperature with stirring over $1 \mathrm{~h}$. Then the mixture was cooled to $0{ }^{\circ} \mathrm{C}$, and the alkynol $(S)$ 17 (3.15 g, $28.1 \mathrm{mmol}$ ) was added dropwise over $20 \mathrm{~min}$. The resulting slurry was warmed to room temperature and stirred at the same temperature. The completion of reaction was monitored by TLC. The mixture was cooled to $0{ }^{\circ} \mathrm{C}$, and ice chips were added slowly and extracted with ether. The combined extracts were washed with $1 \mathrm{~N} \mathrm{HCl}$, saturated aqueous sodium bicarbonate and brine, and then dried $\left(\mathrm{Na}_{2} \mathrm{SO}_{4}\right)$ and concentrated under reduced pressure. The crude residue was purified by flash chromatography $\left(\mathrm{Et}_{2} \mathrm{O} / \mathrm{Hexane} 20-30 \%\right)$ to

give $12(2.71 \mathrm{~g}, 24.2 \mathrm{mmol}, 85 \%)$ as a colorless oil. $R_{f}=0.28(20 \%$ EtOAc/Hexane $):[\alpha]_{\mathrm{D}}{ }^{25}=$ $+8.0\left(c=1.0, \mathrm{CHCl}_{3}\right)$; IR (neat): 3309, 2934, 2862, 2117, 1460, 1432, 1374, 1128, 1093, 937, $821 \mathrm{~cm}^{-1} ;{ }^{1} \mathrm{H}$ NMR $\left(600 \mathrm{MHz}, \mathrm{CDCl}_{3}\right) \delta 3.83(\mathrm{qt}, J=6.0,6.0 \mathrm{~Hz}, 1 \mathrm{H}), 2.23(\mathrm{td}, J=7.2,3.0$ $\mathrm{Hz}, 2 \mathrm{H}), 1.95(\mathrm{t}, J=3.0 \mathrm{~Hz}, 1 \mathrm{H}), 1.67-1.56(\mathrm{~m}, 4 \mathrm{H}), 1.20(\mathrm{~d}, J=6.0 \mathrm{~Hz}, 3 \mathrm{H}) \mathrm{ppm} ;{ }^{13} \mathrm{C} \mathrm{NMR}$ $\left(150 \mathrm{MHz}, \mathrm{CDCl}_{3}\right) \delta 84.3,68.5,67.6,38.2,24.7,23.6,18.4$ ppm. $^{3}$

\section{(S)-tert-Butyl(hept-6-yn-2-yloxy)dimethylsilane, 18}

\footnotetext{
${ }^{3}$ Wu, Y.; Gao, J. Org. Lett. 2008, 10, 1533-1536.
} 


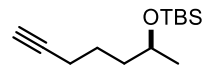

To a stirred solution of $12(1.94 \mathrm{~g}, 17.3 \mathrm{mmol})$ in dimethylformamide $(10 \mathrm{~mL})$ at room temperature was added imidazole $(2.71 \mathrm{~g}, 39.8 \mathrm{mmol})$, followed by tert-butyldimethylsilyl chloride (4.82 g, $32.00 \mathrm{mmol})$ under $\mathrm{N}_{2}$. The resulting mixture was stirred at the same temperature for $16 \mathrm{~h}$. The reaction was quenched by adding water $(10 \mathrm{~mL})$ and extracted with $\mathrm{Et}_{2} \mathrm{O}(3 \times 40 \mathrm{~mL})$. The combined organic layers were washed with brine, dried $\left(\mathrm{Na}_{2} \mathrm{SO}_{4}\right)$, filtered and concentrated under reduced pressure. The crude residue was purified by flash chromatography $(0-1 \%$ EtOAc in hexanes) on silica gel $(100 \mathrm{~mL})$ to afford $(S)$-tert-butyl(hept6-yn-2-yloxy)dimethylsilane (18) (3.88 g, $17.15 \mathrm{mmol}, 99 \%)$ as a colorless oil: $R_{f}=0.29$ (hexanes); $[\alpha]_{\mathrm{D}}{ }^{20}=+6.95\left(c=0.34, \mathrm{CHCl}_{3}\right) ; \quad$ IR (neat): 3315, 2956, 2930, 2858, 2120, 1473 , 1374, $1256 \mathrm{~cm}^{-1} ;{ }^{1} \mathrm{H}$ NMR (500 MHz, $\left.\mathrm{CDCl}_{3}\right) \delta 3.84-3.78(\mathrm{~m}, 1 \mathrm{H}), 2.23-2.15(\mathrm{~m}, 2 \mathrm{H}), 1.94$ $(\mathrm{t}, J=2.5 \mathrm{~Hz}, 1 \mathrm{H}), 1.66-1.47(\mathrm{~m}, 4 \mathrm{H}), 1.14(\mathrm{~d}, J=6 \mathrm{~Hz}, 3 \mathrm{H}), 0.88(\mathrm{~m}, 9 \mathrm{H}), 0.049(\mathrm{~s}, 6 \mathrm{H})$ ppm; ${ }^{13} \mathrm{C}$ NMR $\left(400 \mathrm{MHz}, \mathrm{CDCl}_{3}\right) \delta 84.79,68.40,68.31,38.81,26.07,24.93,23.99,18.66$, 18.31, -4.56. ${ }^{4}$ HRMS (MALDI) calcd for $\mathrm{C}_{13} \mathrm{H}_{26} \mathrm{OSi}^{+}[\mathrm{M}]^{+} 226.1753$, found 226.1752.

\section{Procedure A (Ruthenium catalyzed addition)}

\footnotetext{
${ }^{4}$ Oliveira, R. A.; Oliveira, J. M.; Rahmeier, L. H.; Comasseto, J. V.; Marino, J. P.; Menezes, P. H. Tetrahedron Lett. 2008, 49, 5759-5761.
} 
To a stirred suspension of $\mathrm{Ru}_{3}(\mathrm{CO})_{12}(47.9 \mathrm{mg}, 0.075 \mathrm{mmol})$ in $3.5 \mathrm{~mL}$ NMP added [PPN]Cl (216.5 mg, $0.38 \mathrm{mmol}$ ) under $\mathrm{N}_{2}$ at $100{ }^{\circ} \mathrm{C}$ for 15 mins. Then added tert-butyl-(hex-5-yn-1yloxy)dimethylsilane $(800 \mathrm{mg}, 3.77 \mathrm{mmol})$, followed by ethyl acrylate $(3.5 \mathrm{~mL}, 37.66 \mathrm{mmol}$, 10 eq), the resulting mixture was stirred at $90{ }^{\circ} \mathrm{C}$ for 24 hours. The completion of the reaction was monitored by TLC. The reaction was quenched by adding water and extracted with $\mathrm{Et}_{2} \mathrm{O}$ $(3 \times 100 \mathrm{~mL})$. The combined organic layers were dried over anhydrous $\mathrm{Na}_{2} \mathrm{SO}_{4}$, filtered and concentrated under reduced pressure. The crude residue was purified by silica gel chromatography $\left(0-2 \% \mathrm{Et}_{2} \mathrm{O}\right.$ in hexanes) to afford a mixture of ester $\mathbf{1 5 b}$ and $(2 E, 4 E)-\mathbf{1 6} \mathbf{b}$ (313 $\mathrm{mg}, 1.00 \mathrm{mmol}, 75 \%)$ as a colorless oil with ratio of $15 \mathbf{b} /(2 E, 4 E)-16 \mathbf{b}=2: 1$ determined by ${ }^{1}$ HNMR. Prep-HPLC $(10 \mathrm{~mm} \times 250 \mathrm{~mm})$ was applied to separate the two isomers with $2 \%$ EA: $98 \%$ Hexanes at $2 \mathrm{~mL} / \mathrm{min}$ flowing speed. The reaction also yielded a side product $(2 Z$, $4 E)-16 \mathbf{b}(25 \mathrm{mg}, 0.08 \mathrm{mmol}, 6 \%)$, whose $R_{f}$ value is higher than $15 \mathbf{b}$ and $(2 E, 4 E)-16 \mathbf{b}$ and was easily separated by silica gel column from $15 \mathbf{b} /(2 E, 4 E)-16 \mathbf{b}$ mixture.

\section{Procedure B (specific example for Palladium-NHC methods)}

1,3-Bis(2,4,6-trimethylphenyl)imidazolium chloride (4.8 mg, $0.014 \mathrm{mmol}), \mathrm{Pd}(\mathrm{OAc})_{2}(3.2 \mathrm{mg}$, $0.0141 \mathrm{mmol})$ and $t$-BuOK $(1.9 \mathrm{mg}, 0.017 \mathrm{mmol})$ were added to a sealed tube under argon. Dry THF $(0.8 \mathrm{~mL})$ was injected and the mixture was stirred at room temperature for $2 \mathrm{hr}$, followed by the addition of tert-butyl-(hex-5-yn-1-yloxy)dimethylsilane (100 mg, $0.47 \mathrm{mmol}$ ) and ethyl acrylate $\left(47 \mathrm{mg}, 4.7 \mathrm{mmol}\right.$ ). The mixture was stirred at $60{ }^{\circ} \mathrm{C}$ for $30 \mathrm{~h}$. The solvent was evaporated and the crude reaction mixture was then purified by flash chromatography on silica gel (0-2\% Et ${ }_{2} \mathrm{O}$ in hexanes), which didn't afford the desired product. 


\section{Procedure C (specific example for Palladium-Phosphine methods)}

A mixture of $\mathrm{Pd}(\mathrm{OAc})_{2}(2 \mathrm{mg}, 8.8 \mathrm{umol})$ and $(o \text {-tol })_{3} \mathrm{P}(2.7 \mathrm{mg}, 8.8 \mathrm{umol})$ in $0.5 \mathrm{~mL}$ of dry benzene was stirred for $15 \mathrm{~min}$ at room temperature under argon. Alkyne 18 (50 mg, $0.22 \mathrm{mmol})$ and ethyl acrylate $(220 \mathrm{mg}, 2.2 \mathrm{mmol})$ were added by syringe. After $20 \mathrm{hr}$ of stirring at room temperature, the reaction was concentrated in vacuo and the residue was purified by flash chromatography (0-4\% EtOAc in hexanes) to give mixture of ester 19 and $(2 E, 4 E)-20(11.5$ $\mathrm{mg}, 0.035 \mathrm{mmol}, 16 \%$ ) as a colorless oil.

\section{Ester 15b}

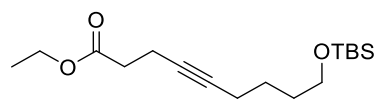

$R_{f}\left(10 \% \mathrm{Et}_{2} \mathrm{O} /\right.$ hexane $)=0.6$; IR (thin film, $\left.\mathrm{cm}^{-1}\right): 2930,2857,2361,1740,1163,1105,837$, 780; ${ }^{1} \mathrm{H}$ NMR (400 MHz, $\left.\mathrm{CDCl}_{3}\right) \delta 4.15(\mathrm{q}, J=7.2 \mathrm{~Hz}, 2 \mathrm{H}), 3.61(\mathrm{t}, J=6.2 \mathrm{~Hz}, 2 \mathrm{H}), 2.47(\mathrm{~m}$, 4H), $2.15(\mathrm{t}, J=7.2 \mathrm{~Hz}, 2 \mathrm{H}), 1.57-1.47(\mathrm{~m}, 4 \mathrm{H}), 1.26(\mathrm{t}, J=7.2 \mathrm{~Hz}, 3 \mathrm{H}), 0.89(\mathrm{~s}, 9 \mathrm{H}), 0.04$ (s, 6H). ${ }^{13} \mathrm{C}$ NMR $\left(100 \mathrm{MHz}, \mathrm{CDCl}_{3}\right) \delta 172.43,81.08,78.51,62.94,60.73,34.38,32.14,26.18$, 25.57, 18.71, 18.57, 15.01, 14.45, -5.07; HRMS (MALDI) calcd for $\mathrm{C}_{17} \mathrm{H}_{33} \mathrm{O}_{3} \mathrm{Si}^{+}[\mathrm{M}+\mathrm{H}]^{+}$ 313.2193, found 313.2195

\section{Ester 16b}




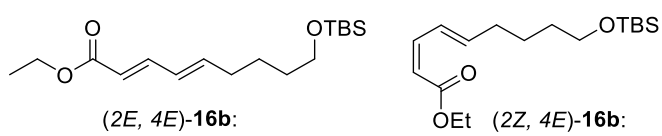

$(2 E, 4 E)-16 \mathbf{b}: R_{f}\left(5 \% \mathrm{Et}_{2} \mathrm{O} /\right.$ hexane $)=0.55 ; \mathrm{IR}\left(\right.$ thin film, $\left.\mathrm{cm}^{-1}\right): 2930,2860,1716,1644,1618$, 1463, 1246, 1138, 1001, 835, 779; ${ }^{1} \mathrm{H}$ NMR $\left(400 \mathrm{MHz}, \mathrm{CDCl}_{3}\right) . \delta 7.24(\mathrm{dd}, J=15.6,9.6 \mathrm{~Hz}$, 1H), $6.17(\mathrm{dd}, J=15.2,9.6 \mathrm{~Hz}, 1 \mathrm{H}), 6.11(\mathrm{dt}, J=15.2,6.4 \mathrm{~Hz}, 1 \mathrm{H}) 5.78(\mathrm{~d}, J=15.6 \mathrm{~Hz}, 1 \mathrm{H})$, $4.19(\mathrm{q}, J=7.2 \mathrm{~Hz}, 2 \mathrm{H}), 3.61(\mathrm{t}, J=6.0 \mathrm{~Hz}, 2 \mathrm{H}), 2.18(\mathrm{dt}, J=6.8,6.4 \mathrm{~Hz}, 2 \mathrm{H}), 1.47-1.53(\mathrm{~m}$, 4H), 1.29 (t, $J=7.2 \mathrm{~Hz}, 3 \mathrm{H}), 0.89(\mathrm{~s}, 9 \mathrm{H}), 0.04(\mathrm{~s}, 6 \mathrm{H}) .{ }^{13} \mathrm{C} \mathrm{NMR}\left(100 \mathrm{MHz}, \mathrm{CDCl}_{3}\right) \delta 167.51$, 145.21, 144.58, 128.71, 119.51, 63.08, 60.38, 32.94, 32.49, 26.18, 25.26, 18.58, 14.53, -5.07.;

$(2 Z, 4 E)-16 b: R_{f}\left(5 \% \mathrm{Et}_{2} \mathrm{O} /\right.$ hexane $)=0.65 ;{ }^{1} \mathrm{H} \mathrm{NMR}\left(400 \mathrm{MHz}, \mathrm{CDCl}_{3}\right) \delta 7.36(\mathrm{dd}, J=14.8$, $11.2 \mathrm{~Hz}, 1 \mathrm{H}), 6.54(\mathrm{dd}, J=11.5,11.4 \mathrm{~Hz}, 1 \mathrm{H}), 6.05(\mathrm{dt}, J=14.8,7.2 \mathrm{~Hz}, 1 \mathrm{H}), 5.56(\mathrm{~d}, J=11.6$ $\mathrm{Hz}, 1 \mathrm{H}), 4.18(\mathrm{dd}, J=14.2,7.1 \mathrm{~Hz}, 2 \mathrm{H}), 3.61(\mathrm{t}, J=6.2 \mathrm{~Hz}, 2 \mathrm{H}), 2.22(\mathrm{dt}, J=7.2,6.8 \mathrm{~Hz}, 2 \mathrm{H})$, $1.50(\mathrm{dd}, J=6.8,3.7 \mathrm{~Hz}, 4 \mathrm{H}), 1.29(\mathrm{t}, J=5.0 \mathrm{~Hz}, 3 \mathrm{H}), 0.89$ (s, 9H), 0.04 (s, 6H). HRMS (MALDI) calcd for $\mathrm{C}_{17} \mathrm{H}_{33} \mathrm{O}_{3} \mathrm{Si}^{+}[\mathrm{M}+\mathrm{H}]^{+}$313.2193, found 313.2190

\section{Ester 15a}




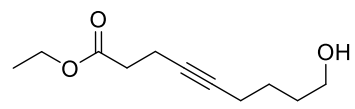

$R_{f}(100 \%$ EtOAc $)=0.65 ;$ IR $\left(\right.$ thin film, $\left.\mathrm{cm}^{-1}\right): 3353,2919,2360,2342,1734,1163,1043 ;{ }^{1} \mathrm{H}$ $\operatorname{NMR}\left(400 \mathrm{MHz}, \mathrm{CDCl}_{3}\right) \delta 4.15(\mathrm{q}, J=7.2 \mathrm{~Hz}, 2 \mathrm{H}), 3.66(\mathrm{t}, J=6.4 \mathrm{~Hz}, 2 \mathrm{H}), 2.44-2.52(\mathrm{~m}$, $4 \mathrm{H}), 2.18(\mathrm{t}, J=7.2 \mathrm{~Hz}, 2 \mathrm{H}), 1.65(\mathrm{~m}, 2 \mathrm{H}), 1.56(\mathrm{~m}, 2 \mathrm{H}), 1.25(\mathrm{t}, J=7.2 \mathrm{~Hz}, 3 \mathrm{H}) .{ }^{13} \mathrm{C} \mathrm{NMR}$ $\left(100 \mathrm{MHz}, \mathrm{CDCl}_{3}\right) \delta 172.25,80.89,78.82,62.69,60.77,34.36,32.01,25.31,18.67,15.01$, 14.45. HRMS (MALDI) calcd for $\mathrm{C}_{11} \mathrm{H}_{18} \mathrm{O}_{3} \mathrm{Na}^{+}[\mathrm{M}+\mathrm{Na}]^{+}$221.1143, found 221.1148.

\section{Ester 19,}

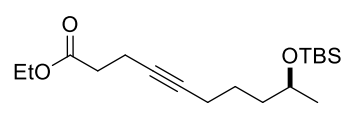

$R_{f}\left(5 \% \mathrm{Et}_{2} \mathrm{O} /\right.$ hexane $)=0.48 ;[\alpha]_{\mathrm{D}}^{20}=+3.46\left(\mathrm{CHCl}_{3}, \mathrm{c}=0.35\right)$ IR $\left(\right.$ thin film, $\left.\mathrm{cm}^{-1}\right) 2929,2856$, 2361, 2342, 1740, 1374, 1255, 1163, 1093, $836 \mathrm{~cm}^{-1} ;{ }^{1} \mathrm{H}$ NMR (400 MHz, $\left.\mathrm{CDCl}_{3}\right) \delta 4.15$ (q, $J$ $=7.1 \mathrm{~Hz}, 2 \mathrm{H}), 3.79\left(\mathrm{qt}, J_{1}=J_{2}=6.0 \mathrm{~Hz}, 1 \mathrm{H}\right), 2.48(\mathrm{~m}, 4 \mathrm{H}), 2.12(\mathrm{t}, J=5.9 \mathrm{~Hz}, 2 \mathrm{H}), 1.53-1.41$ $(\mathrm{m}, 5 \mathrm{H}), 1.26(\mathrm{t}, J=7.1 \mathrm{~Hz}, 3 \mathrm{H}), 1.12(\mathrm{~d}, J=6.0 \mathrm{~Hz}, 3 \mathrm{H}), 0.88(\mathrm{~s}, 9 \mathrm{H}), 0.04(\mathrm{~s}, 6 \mathrm{H}) .{ }^{13} \mathrm{C} \mathrm{NMR}$ $\left(100 \mathrm{MHz}, \mathrm{CDCl}_{3}\right) \delta 172.40,81.14,78.47,68.44,60.71,38.96,34.39,26.12,25.39,23.99$, 18.98, 18.36, 15.01, 14.45, -4.18. EI-HRMS calcd for $\mathrm{C}_{18} \mathrm{H}_{34} \mathrm{O}_{3} \mathrm{Si}^{+}[\mathrm{M}]^{+}:$326.2277. Found 326.2281 .

\section{Ester 20}




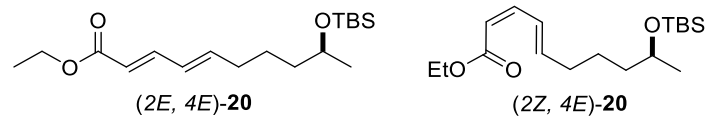

$(2 E, 4 E)-20: R_{f}\left(5 \% \mathrm{Et}_{2} \mathrm{O} /\right.$ hexane $)=0.5 ; \mathrm{IR}\left(\right.$ thin film, $\left.\mathrm{cm}^{-1}\right): 2930,2857,1716,1644,1463$, 1246, 1138, 1037, 1001, 835; ${ }^{1} \mathrm{H}$ NMR $\left(400 \mathrm{MHz}, \mathrm{CDCl}_{3}\right) \delta 7.25(\mathrm{dd}, J=15.6,10.0 \mathrm{~Hz}, 1 \mathrm{H})$, $6.16(\mathrm{dd}, J=15.2,10.0 \mathrm{~Hz}, 1 \mathrm{H}), 6.12(\mathrm{dt}, J=15.2,6.5 \mathrm{~Hz}, 2 \mathrm{H}), 5.78(\mathrm{~d}, J=15.6 \mathrm{~Hz}, 1 \mathrm{H}), 4.19$ $(\mathrm{q}, J=7.1 \mathrm{~Hz}, 2 \mathrm{H}), 3.78\left(\mathrm{qt}, J_{1}=J_{2}=6.0 \mathrm{~Hz}, 1 \mathrm{H}\right), 2.16(\mathrm{td}, J=6.4,6.0 \mathrm{~Hz}, 2 \mathrm{H}), 1.54-1.37$ $(\mathrm{m}, 4 \mathrm{H}), 1.29(\mathrm{t}, J=7.1 \mathrm{~Hz}, 3 \mathrm{H}), 1.11(\mathrm{~d}, J=6.1 \mathrm{~Hz}, 3 \mathrm{H}), 0.88(\mathrm{~s}, 9 \mathrm{H}), 0.04(\mathrm{~s}, 6 \mathrm{H}) ;{ }^{13} \mathrm{C} \mathrm{NMR}$ $\left(100 \mathrm{MHz}, \mathrm{CDCl}_{3}\right) \delta 167.52,145.24,144.67,128.68,119.49,68.54,60.38,39.34,33.19,26.11$, $25.10,24.04,18.36,14.53,-4.50 .{ }^{5}(2 Z, 4 E)-20: R_{f}\left(5 \% \mathrm{Et}_{2} \mathrm{O} /\right.$ hexane $)=0.6 ;{ }^{1} \mathrm{H}$ NMR $(400$ $\left.\mathrm{MHz}, \mathrm{CDCl}_{3}\right) \delta 7.34(\mathrm{dd}, J=15.2,12.0 \mathrm{~Hz}, 1 \mathrm{H}), 6.55\left(\mathrm{dd}, J_{1}=J_{2}=11.4 \mathrm{~Hz}, 1 \mathrm{H}\right), 6.04(\mathrm{dt}, J$ $=14.8,7.2 \mathrm{~Hz}, 1 \mathrm{H}), 5.56(\mathrm{~d}, J=11.6 \mathrm{~Hz}, 1 \mathrm{H}), 4.18(\mathrm{~d}, J=7.2 \mathrm{~Hz}, 2 \mathrm{H}), 3.77(\mathrm{~d}, J=11.5 \mathrm{~Hz}$, 1H), $2.17(\mathrm{dt}, \mathrm{J}=7.2,6.8 \mathrm{~Hz}, 2 \mathrm{H}), 1.42(\mathrm{~s}, 4 \mathrm{H}), 1.28(\mathrm{~d}, J=7.1 \mathrm{~Hz}, 3 \mathrm{H}), 1.11(\mathrm{~d}, J=6.1 \mathrm{~Hz}$, 3H), $0.88(\mathrm{~s}, 9 \mathrm{H}), 0.04(\mathrm{~s}, 6 \mathrm{H}) .{ }^{6} \mathrm{HRMS}(\mathrm{MALDI})$ calcd for $\mathrm{C}_{18} \mathrm{H}_{34} \mathrm{O}_{3} \mathrm{SiNa}^{+}[\mathrm{M}+\mathrm{Na}]^{+}$: 349.2169. Found 349.2169.

\footnotetext{
${ }^{5}$ Mondol, M. M.; Kim, J. H.; Lee, M. A.; Tareq, F. S.; Lee, H. S.,; Lee, Y. J.; Shin, H. J. Nat. Prod. 2011, 74, 1606-1612 6 [a] Schmidt, B.; Kunz, O. Euro. J. Org. Chem. 2012, 5, 1008-1018. [b] Tsuda, M.; Mugishima, T.; Komatsu, K.; Sone, T.; Tanaka, M.; Mikami, Y.; Kobayashi, J. I. J. Nat. Prod. 2003. 66, 412-415.
} 


\section{Ethyl (S)-9-hydroxydec-4-ynoate, 11}

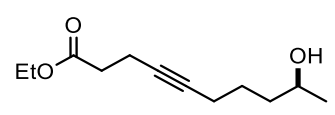

Alkynyl ester 19 (250 mg, $0.766 \mathrm{mmol})$ was dissolved in TBAF solution in THF (4 mL, $1 \mathrm{M})$.

The mixture was stirred at room temperature overnight. The reaction was quenched with water $(10 \mathrm{~mL})$ and extracted with $\mathrm{Et}_{2} \mathrm{O}(3 \times 15 \mathrm{~mL})$. The combine organic layer was dried over anhydrous $\mathrm{Na}_{2} \mathrm{SO}_{4}$, filtered and concentrated under reduced pressure. The crude residue was purified by column chromatography ( $20 \% \mathrm{Et}_{2} \mathrm{O}$ in hexane) to afford alkynyl ester $\mathbf{1 1}(154 \mathrm{mg}$, $0.73 \mathrm{mmol}, 95 \%)$ as colorless oil. $R_{f}=0.22(25 \%$ EtOAc/hexane $) ; \quad[\alpha]_{\mathrm{D}}^{20}=+7.9(c=1.17$, $\mathrm{CHCl}_{3}$ ); IR (neat): 3396, 2927, 2360, 1736, 1550, 1372, 1160, $808 \mathrm{~cm}^{-1} ;{ }^{1} \mathrm{H}$ NMR (400 MHz, $\left.\mathrm{CDCl}_{3}\right) \delta 4.15(\mathrm{q}, J=7.2 \mathrm{~Hz}, 2 \mathrm{H}), 3.82\left(\mathrm{qt}, J_{1}=J_{2}=6.0 \mathrm{~Hz}, 1 \mathrm{H}\right), 2.48(\mathrm{t}, J=\mathrm{Hz}, 2 \mathrm{H}), 2.16(\mathrm{t}$, $J=\mathrm{Hz}, 2 \mathrm{H}), 1.43-1.60(\mathrm{~m}, 6 \mathrm{H}), 1.26(\mathrm{t}, J=7.1 \mathrm{~Hz}, 3 \mathrm{H}), 1.20(\mathrm{~d}, J=6.2 \mathrm{~Hz}, 3 \mathrm{H}) \mathrm{ppm} ;{ }^{13} \mathrm{C}$ NMR $\left(100 \mathrm{MHz}, \mathrm{CDCl}_{3}\right) \delta 172.53,80.97,78.76,67.75,60.92,38.68,34.49,25.44,23.70$, 19.07, 15.18, 14.40 ppm; HRMS (EI) calcd for $\mathrm{C}_{12} \mathrm{H}_{21} \mathrm{O}_{3}{ }^{+}[\mathrm{M}+\mathrm{H}]^{+}: 213.1485$, found 213.1497.

\section{Alkenyl ester 21}




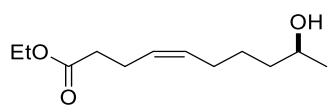

To a stirred solution of alkynyl ester 11 (50 mg, $0.24 \mathrm{mmol})$ in $\mathrm{MeOH}$ was added $\mathrm{Pd} / \mathrm{CaCO}_{3}$ $(5 \%, 50 \mathrm{mg}, 24 \mu \mathrm{mol})$ and quinoline $(12 \mathrm{uL}, 60 \mu \mathrm{mol})$. The resulting mixture was stirred at room temperature under atmosphere for $5 \mathrm{~min}$ and then under $\mathrm{H}_{2}$ for $4 \mathrm{~h}$. The reaction mixture was filtered through Celite and rinsed with $\mathrm{Et}_{2} \mathrm{O}$. The filtrate was extracted with $\mathrm{Et}_{2} \mathrm{O}(3 \times 15$ $\mathrm{mL}$ ). The combine organic layer was dried over anhydrous $\mathrm{Na}_{2} \mathrm{SO}_{4}$, filtered and concentrated under reduced pressure. The crude residue was purified by column chromatography (10-20\% $\mathrm{Et}_{2} \mathrm{O}$ in hexane) to afford alkenyl ester 21 (43 mg, $\left.0.20 \mathrm{mmol}, 85 \%\right)$ as colorless oil. $R_{f}=0.27$ (25\% EtOAc/hexane); $[\alpha]_{\mathrm{D}}{ }^{20}=+5.21\left(c=0.38, \mathrm{CHCl}_{3}\right)$; IR (neat): 3396, 2927, 1736, 1505, 1495, 1372, 1160, $908 \mathrm{~cm}^{-1}$; ${ }^{1} \mathrm{H}$ NMR (400 MHz, $\left.\mathrm{CDCl}_{3}\right) \delta 5.49-5.27(\mathrm{~m}, 2 \mathrm{H}), 4.12(\mathrm{q}, J=$ $7.2 \mathrm{~Hz}, 2 \mathrm{H}), 3.86-3.74(\mathrm{~m}, 1 \mathrm{H}), 2.32-2.34(\mathrm{~m}, 4 \mathrm{H}), 2.13-2.02(\mathrm{~m}, 2 \mathrm{H}), 1.40-1.47(\mathrm{~m}, 4 \mathrm{H})$, $1.25(\mathrm{t}, J=7.1 \mathrm{~Hz}, 3 \mathrm{H}), 1.19(\mathrm{~d}, J=6.4 \mathrm{~Hz}, 3 \mathrm{H}) \mathrm{ppm} ;{ }^{13} \mathrm{C} \mathrm{NMR}\left(100 \mathrm{MHz}, \mathrm{CDCl}_{3}\right) \delta 173.53$, $131.27,128.00,68.14,60.59,39.03,34.57,27.26,25.95,23.73,23.04,14.48$. HRMS (MALDI) calcd for $\mathrm{C}_{12} \mathrm{H}_{22} \mathrm{O}_{3}{ }^{+}[\mathrm{M}]^{+}:$14.1569, found 214.1568.

\section{Carboxylic acid 10}<smiles>CC(O)CCCCCCC(=O)O</smiles> 
To a stirred solution of alkenyl ester 21 (230 mg, $1.1 \mathrm{mmol})$ in $12.5 \mathrm{~mL} \mathrm{THF} / \mathrm{H}_{2} \mathrm{O}$ (3:1) was added $\mathrm{NaOH}(0.86 \mathrm{~g}, 21.5 \mathrm{mmol})$. The reaction was stirred at room temperature for $3 \mathrm{~d}$. The reaction mixture was acidified by $20 \% \mathrm{HCl}$ aq solution and extracted with $\mathrm{Et}_{2} \mathrm{O}(3 \times 30 \mathrm{~mL})$. The combined organic layers was washed with brine and dried over over anhydrous $\mathrm{Na}_{2} \mathrm{SO}_{4}$, filtered and concentrated under reduced pressure. The crude residue was purified by column chromatography (40\% $\mathrm{Et}_{2} \mathrm{O}$ in hexane) to afford carboxylic acid $\mathbf{1 0}(180 \mathrm{mg}, 0.96 \mathrm{mmol}, 90 \%)$ as colorless oil. $R_{f}=0.49\left(100 \%\right.$ EtOAc); $[\alpha]_{\mathrm{D}}{ }^{20}=+1.4\left(\mathrm{CHCl}_{3}, c=0.71\right)$; IR (neat): 3391, 2929, $1710,1267,1140,950 \mathrm{~cm}^{-1} ;{ }^{1} \mathrm{H}$ NMR $\left(400 \mathrm{MHz}, \mathrm{CDCl}_{3}\right) \delta 5.40-5.46(\mathrm{~m}, 1 \mathrm{H}), 5.32-5.38$ (m, 1H), 4.15-4.45 (br, OH), $3.81(\mathrm{~m}, 1 \mathrm{H}), 2.33-2.39(\mathrm{~m}, 4 \mathrm{H}), 2.00-2.15(\mathrm{~m}, 2 \mathrm{H})$, $1.36-1.45(\mathrm{~m}, 4 \mathrm{H}), 1.18(\mathrm{~d}, J=5.9 \mathrm{~Hz}, 3 \mathrm{H}) \mathrm{ppm} ;{ }^{13} \mathrm{C} \mathrm{NMR}\left(100 \mathrm{MHz}, \mathrm{CDCl}_{3}\right) \delta 178.25$, 131.64, 127.66, 68.20, 38.87, 34.29, 27.20, 25.91, 23.61, 22.93. ppm; HRMS (ES) calcd for $\mathrm{C}_{10} \mathrm{H}_{17} \mathrm{O}_{3}^{-}[\mathrm{M}-\mathrm{H}]^{-}:$185.1178, found 185.1181 .

\section{(S)-Phracantholide J, 1}

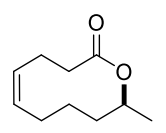


To a stirred solution of arboxylic acid $10(30 \mathrm{mg}, 0.16 \mathrm{mmol})$ in THF $(2 \mathrm{~mL})$ was added $\mathrm{Et}_{3} \mathrm{~N}$ (27 uL, $0.19 \mathrm{mmol}$ ) and 2,4,6-trichlobenzoylchloride (47 mg, $0.19 \mathrm{mmol})$. After stirring for 2 $\mathrm{h}$ at room temperature, the precipitate from reaction was filtered out by Celite and the Celite pad was rinsed with few drops of toluene. The filtrate was diluted with $20 \mathrm{~mL}$ toluene. To make a diluted solution, DMAP (60 mg, $0.5 \mathrm{mmol})$ was dissolved in $170 \mathrm{~mL}$ toluene and then refluxed at $110^{\circ} \mathrm{C}$. The filtrate solution was added to the refluxing DMAP solution dropwise using syringe via a course of $1.5 \mathrm{~h}$. The reaction was finished after $24 \mathrm{~h}$, monitored by TLC. Then cooled the reaction to room temperature and extracted with water and $\mathrm{Et}_{2} \mathrm{O}$. The combined organic layers was dried $\left(\mathrm{Na}_{2} \mathrm{SO}_{4}\right)$, filtered and concentrated under reduced pressure. The crude residue was purified by column chromatography $\left(5 \% \mathrm{Et}_{2} \mathrm{O}\right.$ in hexane) to afford $(S)$ Phracantholide J (1) $(13 \mathrm{mg}, 50 \%)$ as colorless oil. $R_{f}=0.6(20 \%$ EtOAc/hexane $) ;[\alpha]_{\mathrm{D}}{ }^{20}=$ $+28.4\left(c=0.6, \mathrm{CHCl}_{3}\right)\left(\right.$ lit. $=+35.1\left(22^{\circ} \mathrm{C}\right) ;$ IR (neat) 2960, 2926, 1737, 1657, 1650, 1563, $1545,1510,1461,1263,1119 \mathrm{~cm}^{-1} ;{ }^{1} \mathrm{H}$ NMR $\left(400 \mathrm{MHz}, \mathrm{CDCl}_{3}\right) \delta 5.46(\operatorname{tdd}, J=10.4,5.2,1.6$ $\mathrm{Hz}, 1 \mathrm{H}), 5.36(\mathrm{td}, J=11.3,3.9 \mathrm{~Hz}, 1 \mathrm{H}), 5.10(\mathrm{qdd}, J=6.4,4.4,2.0 \mathrm{~Hz}, 1 \mathrm{H}), 2.76(\mathrm{ddd}, J=$ 12.8, 11.2, 4.4 Hz, 1H), 2.67 (d, $J=12.0, \mathrm{~Hz}, 1 \mathrm{H}), 2.53$ (dt, $J=14.4,4.4 \mathrm{~Hz}, 1 \mathrm{H}), 2.22$ (ddd, $J$ $=14.4,12.8,4.4 \mathrm{~Hz}, 1 \mathrm{H}), 1.91(\mathrm{~m}, 4 \mathrm{H}), 1.47-1.41(\mathrm{~m}, 2 \mathrm{H}), 1.25(\mathrm{~d}, J=6.8 \mathrm{~Hz}, 3 \mathrm{H}) ;{ }^{13} \mathrm{C}$ NMR $\left(100 \mathrm{MHz}, \mathrm{CDCl}_{3}\right) \delta 172.13,132.24,127.10,70.94,34.90,31.58,29.70,25.63,22.65$, 22.44, 17.38. ppm; EI -HRMS calcd for $\mathrm{C}_{10} \mathrm{H}_{16} \mathrm{O}_{2}{ }^{+}[\mathrm{M}]^{+}:$168.1150, found 168.1147.

\section{Section C: ${ }^{1} \mathrm{H}$ and ${ }^{13} \mathrm{C}$ NMR spectra}



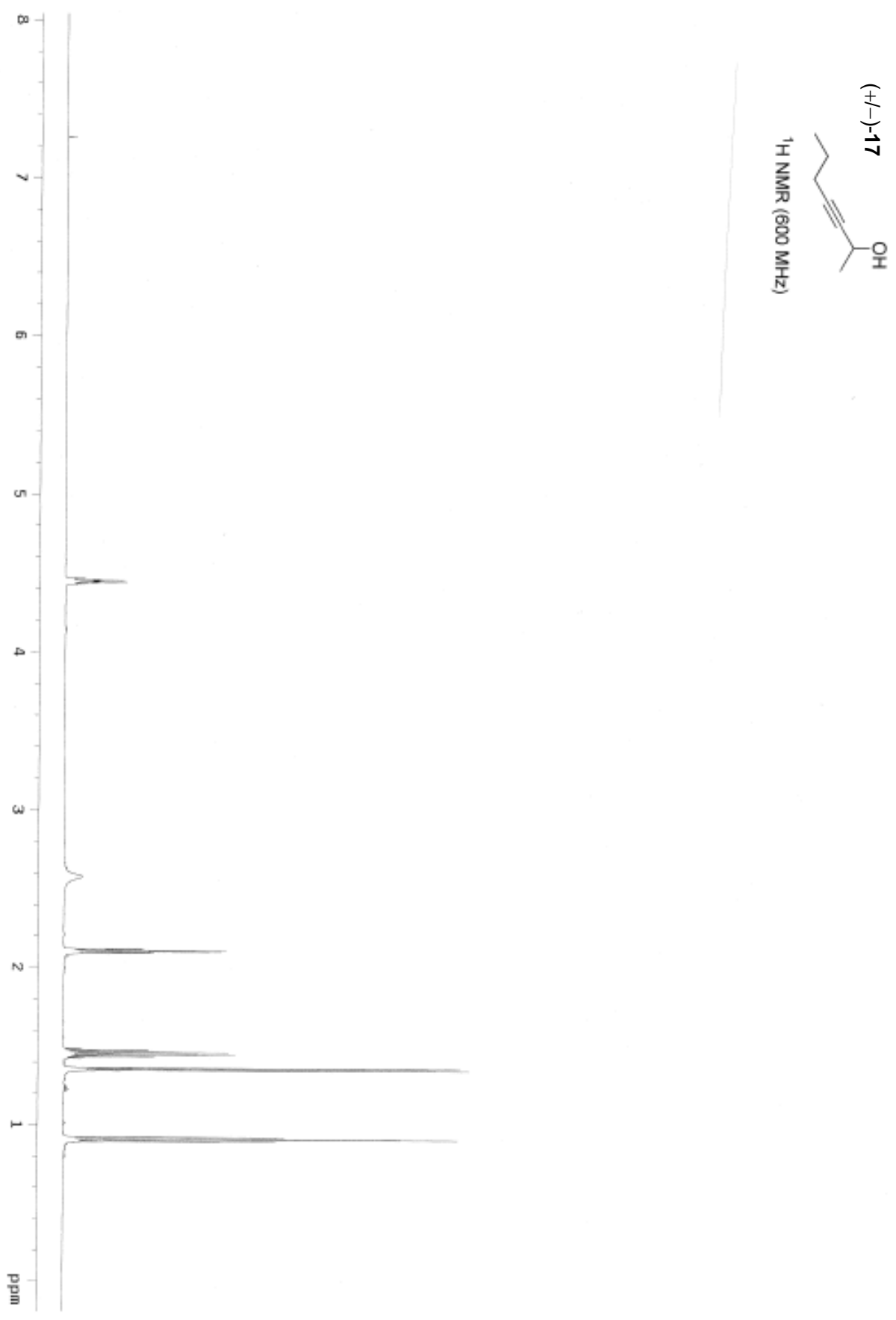

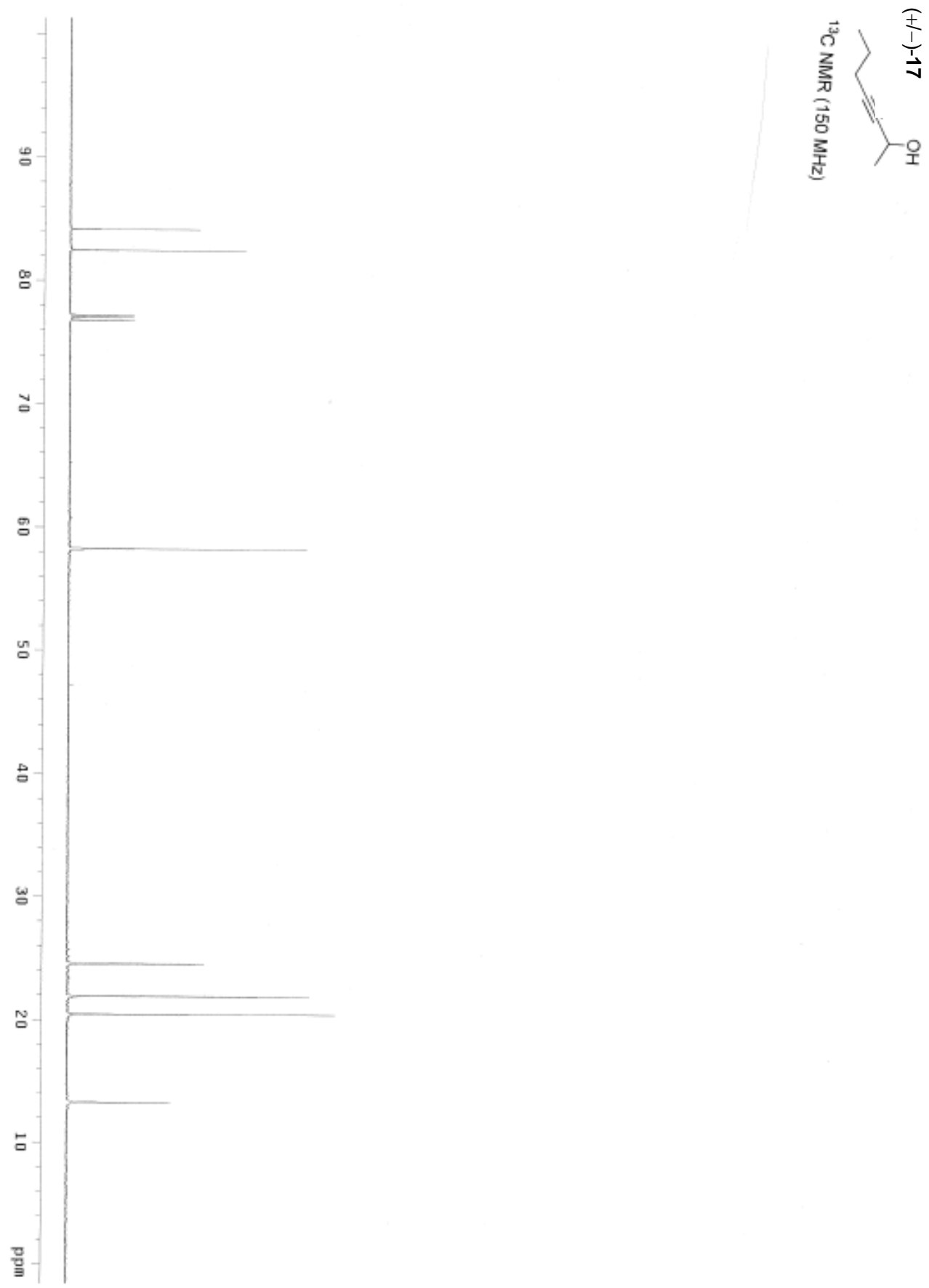

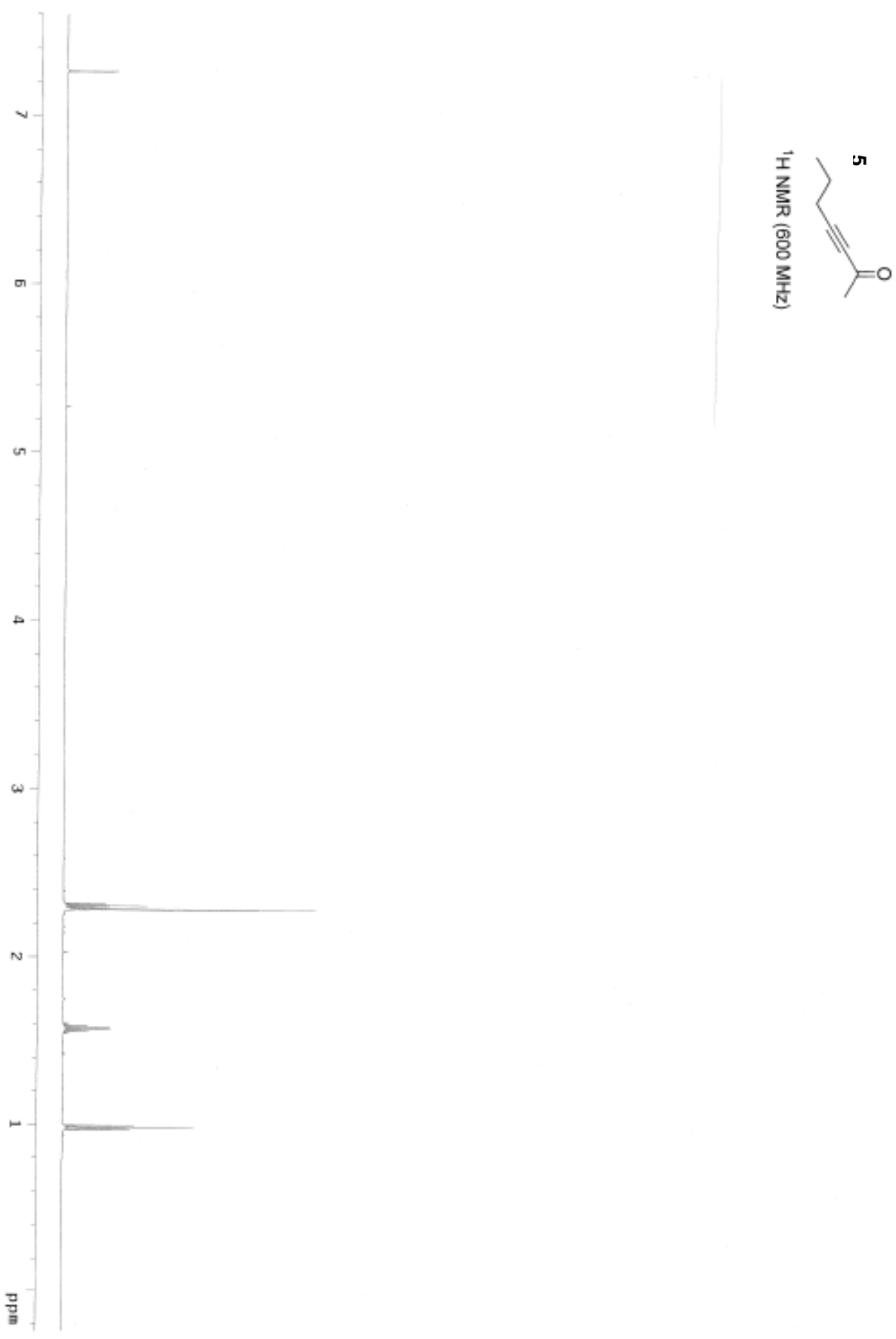

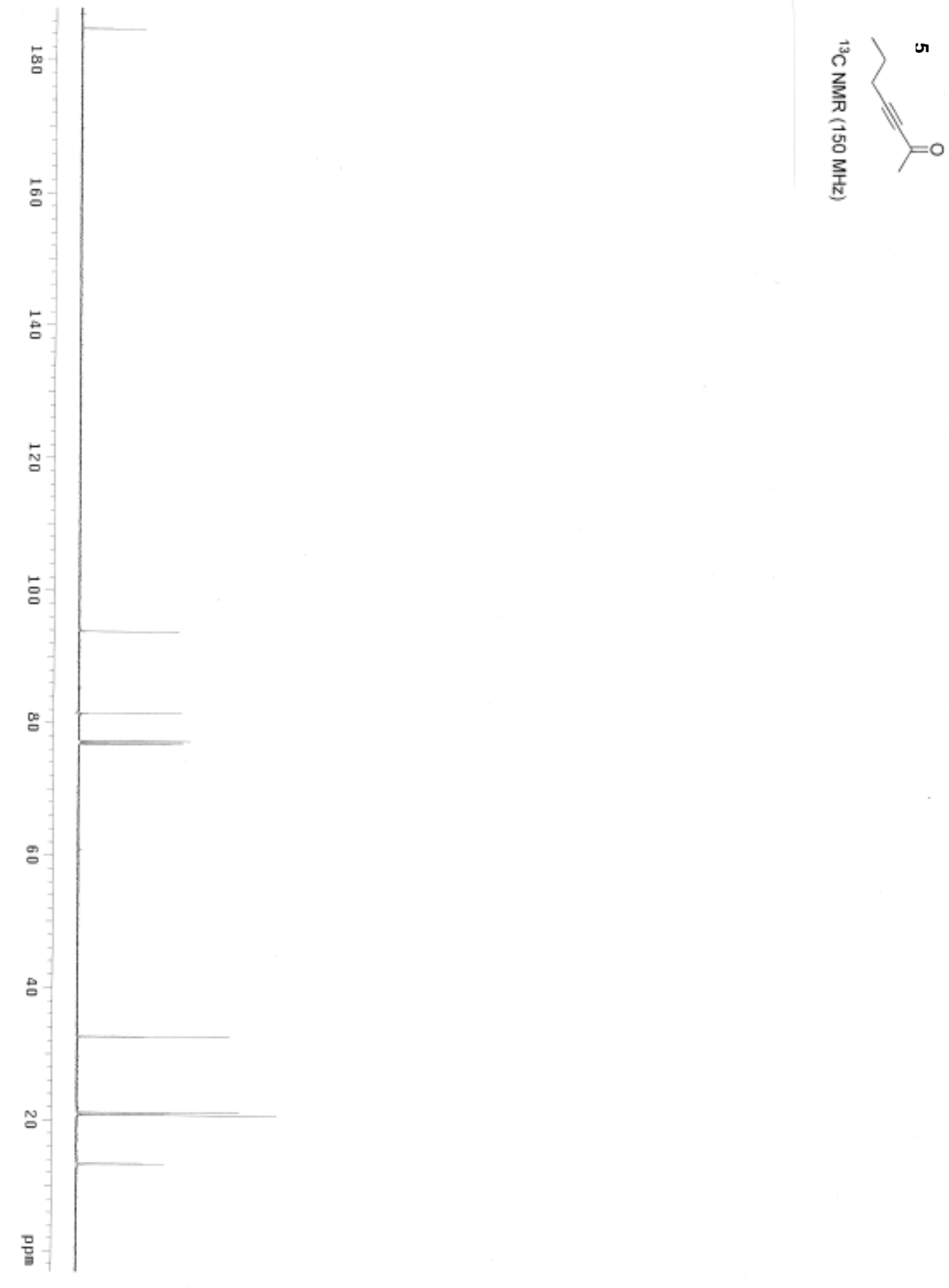

$+$ 


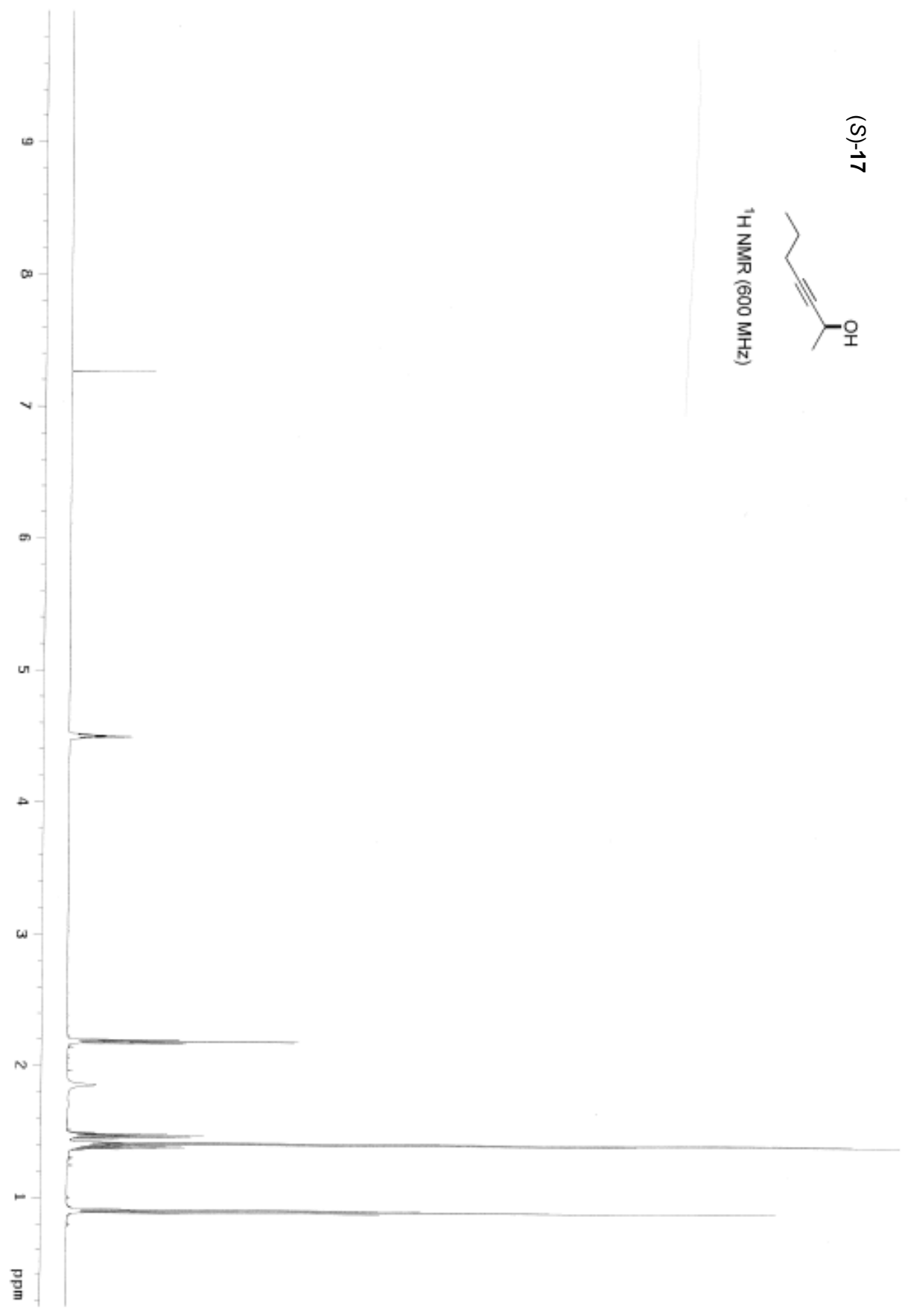




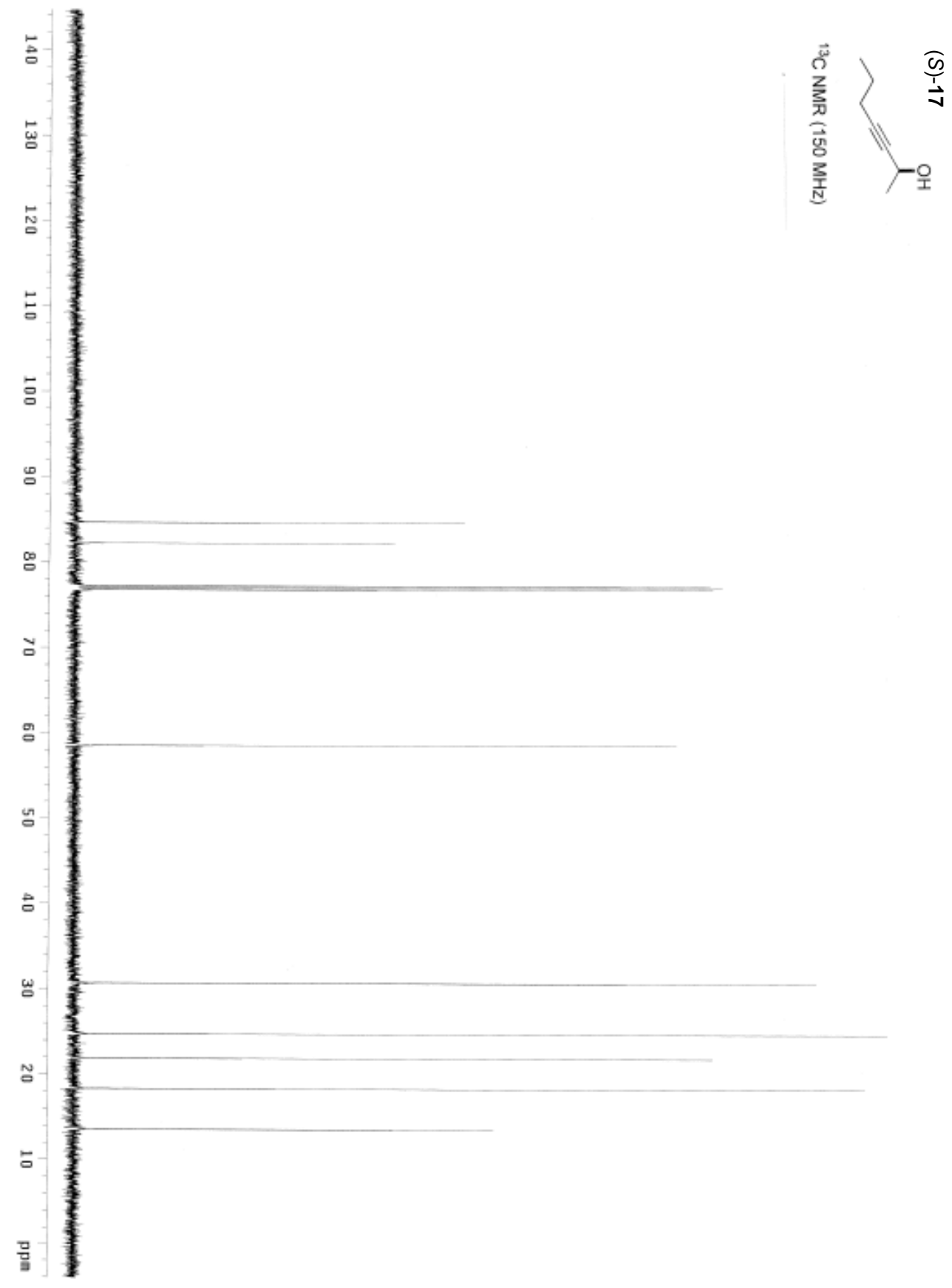




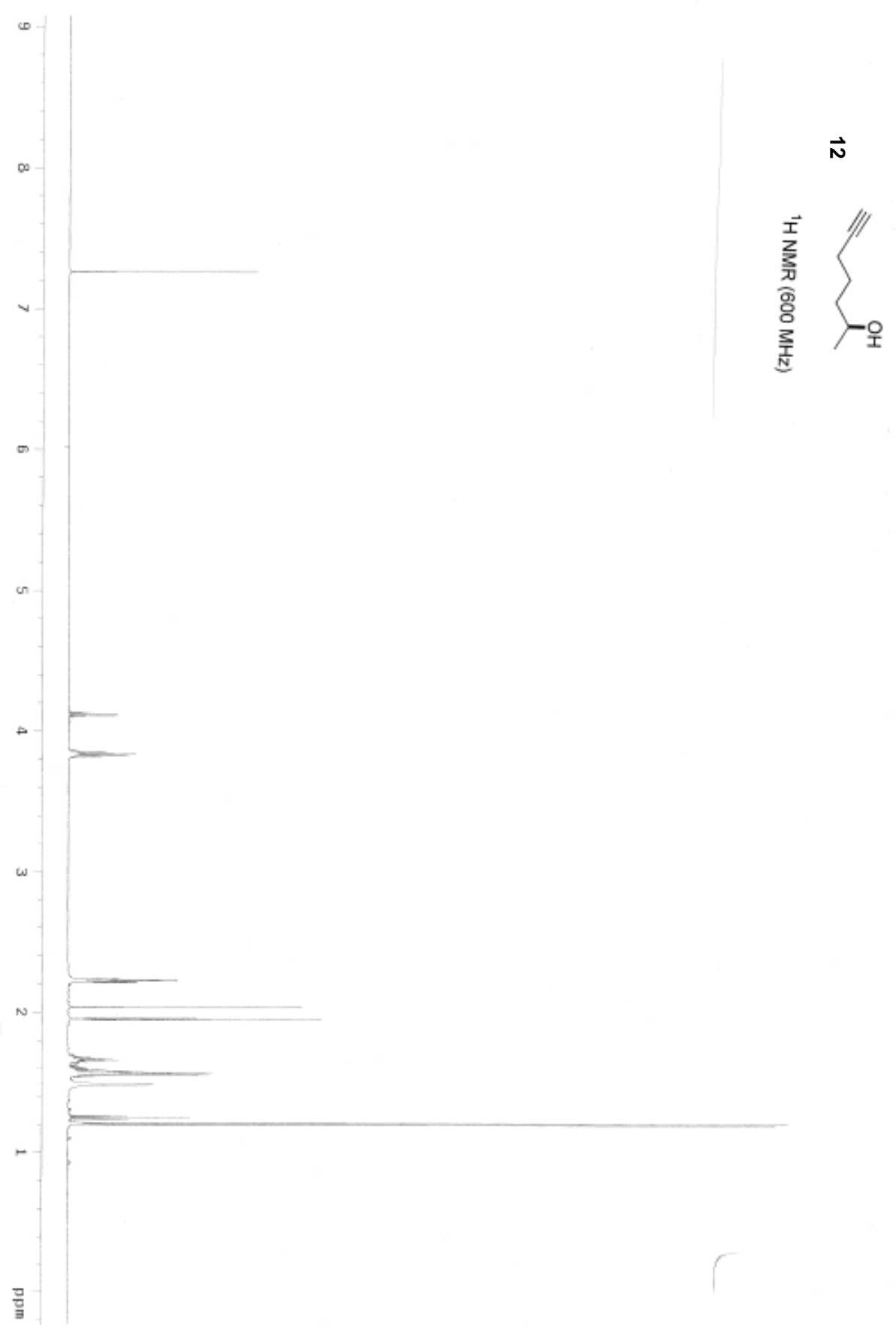




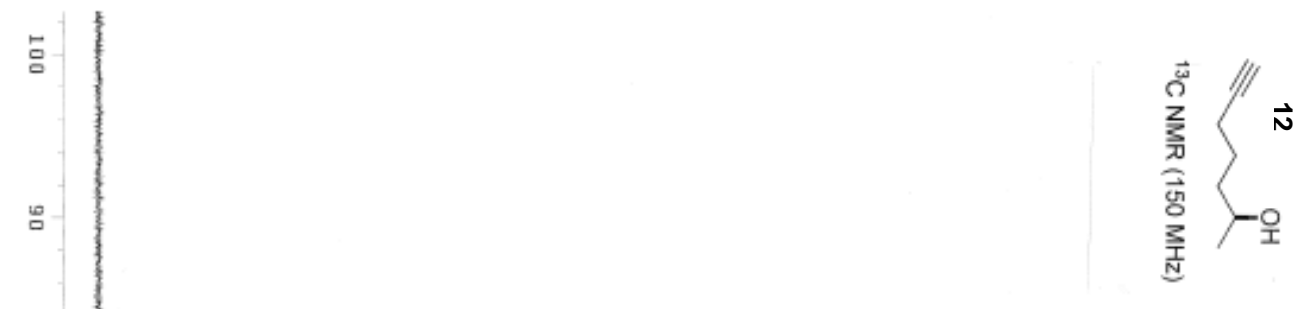

电

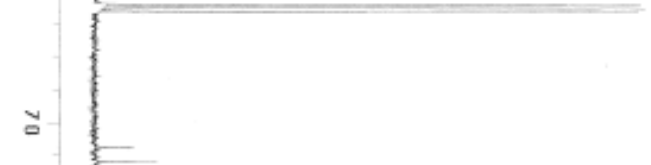

g

แ

b

ผ

N-

5

$5-1$

콜 


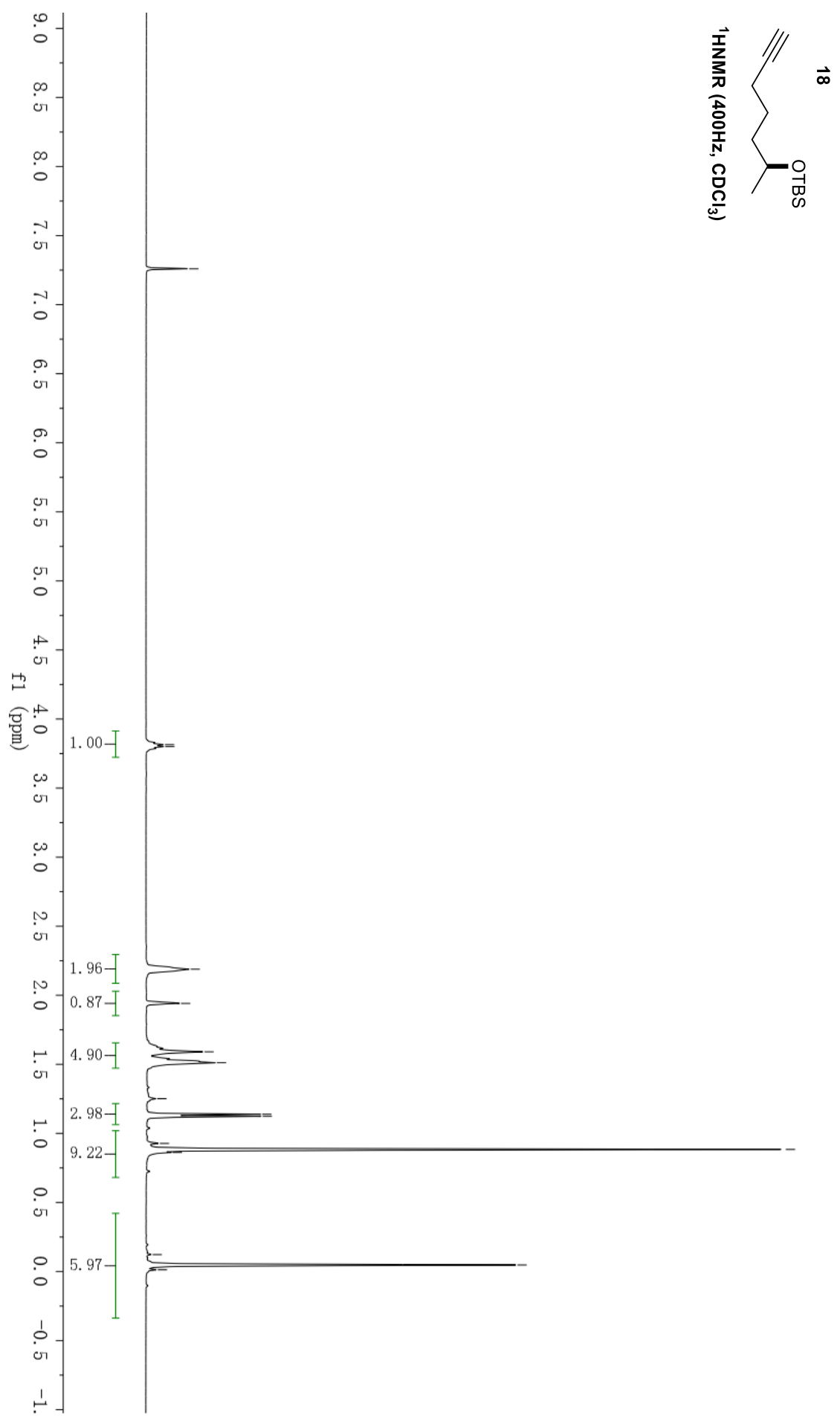

$-7.26$

$<_{3.80}^{3.81}$

$-2.19$

$-1.94$

-1.59
-1.51

1. 25

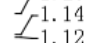

0.93
-0.88

ᄂ. 86

0.12
-0.05

$\chi_{0.01}$ 


$$
\text { H: }
$$




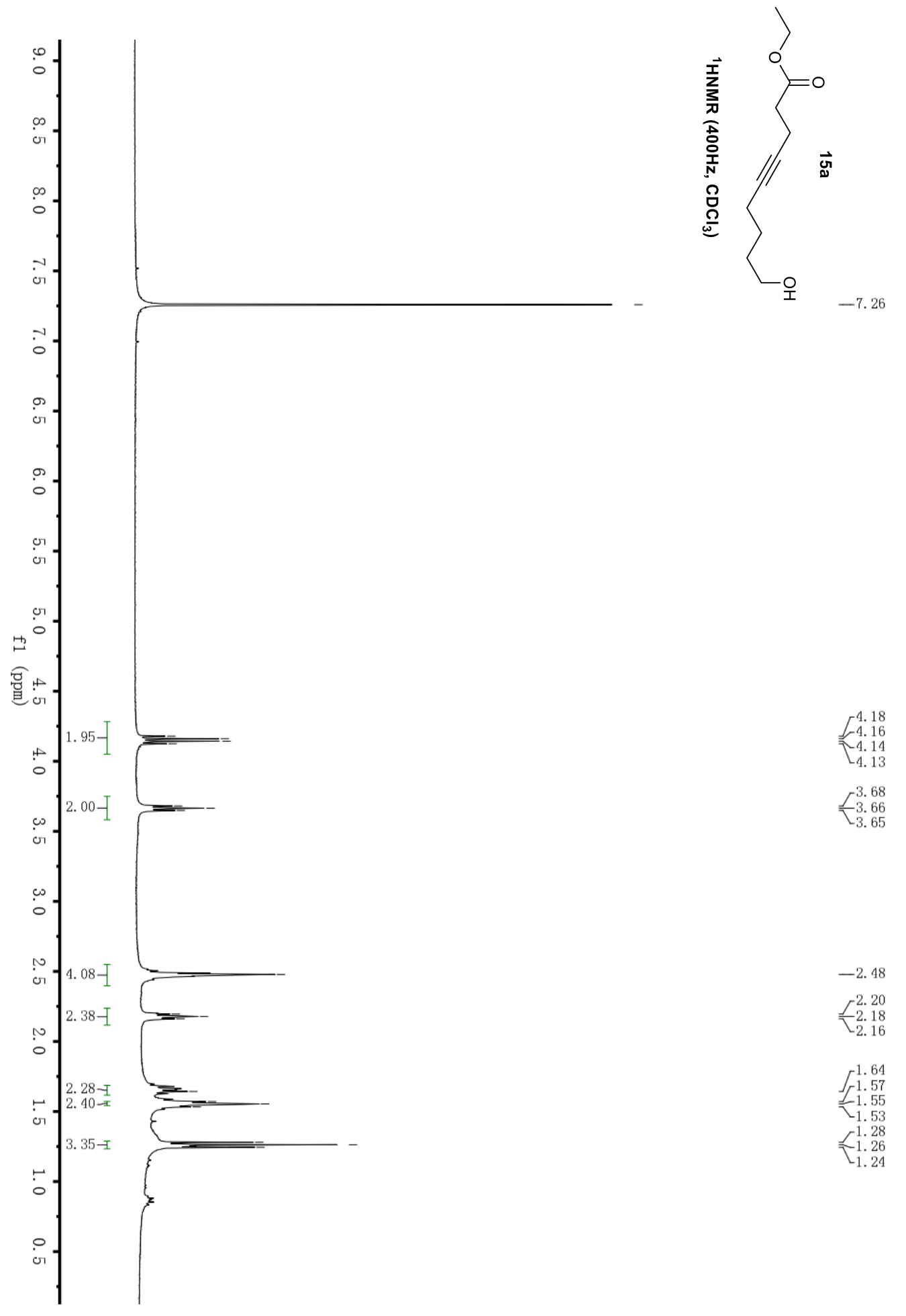




$$
1
$$



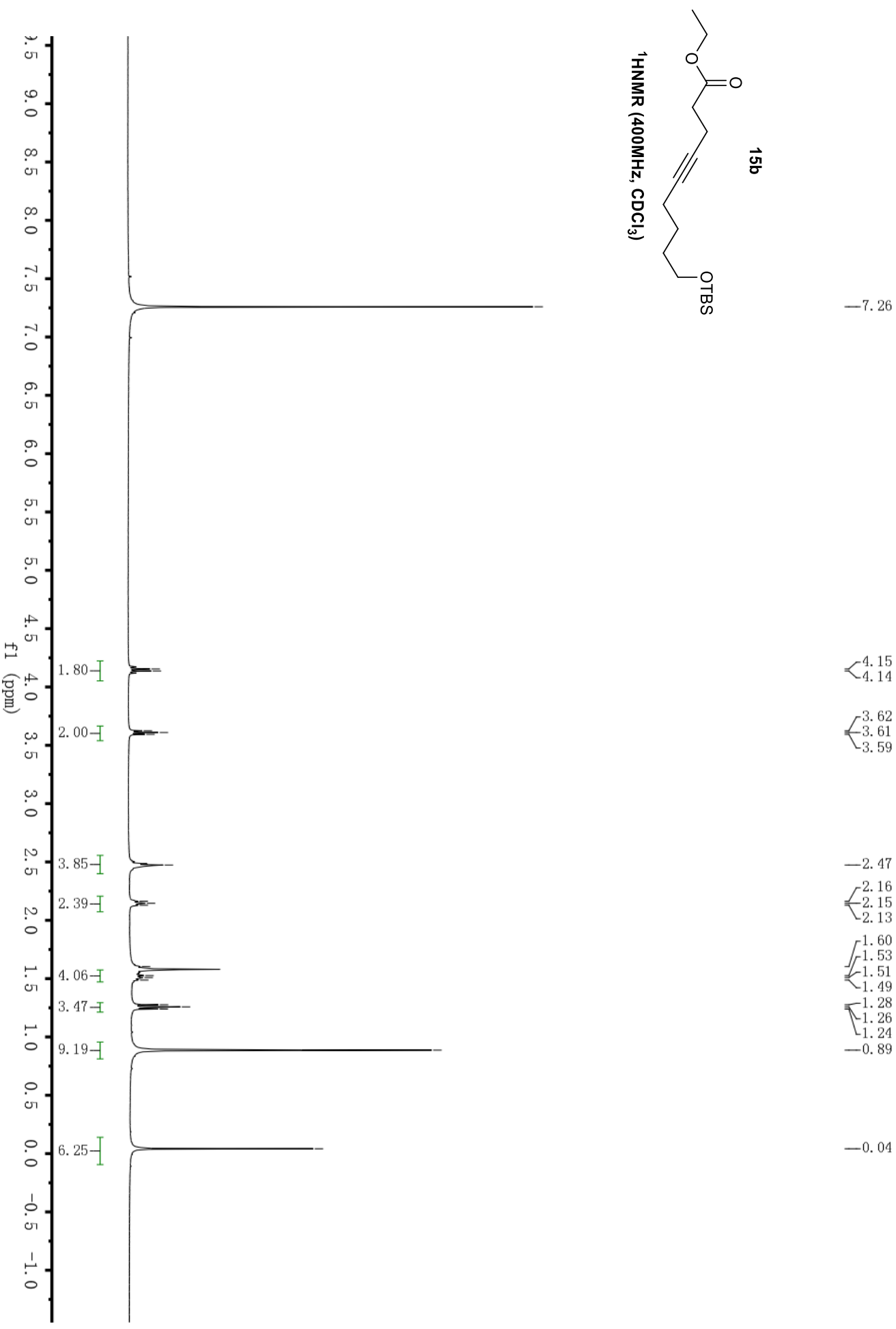

3.62
-3.61
3.59

$-2.47$

2.16
-2.15

$-2.13$

1. 60

$=\begin{aligned} & 1.53 \\ & -1.51 \\ & -1.49\end{aligned}$

$\nwarrow_{-1.26}^{1.28}$

1.24
-0.89 


$$
1
$$



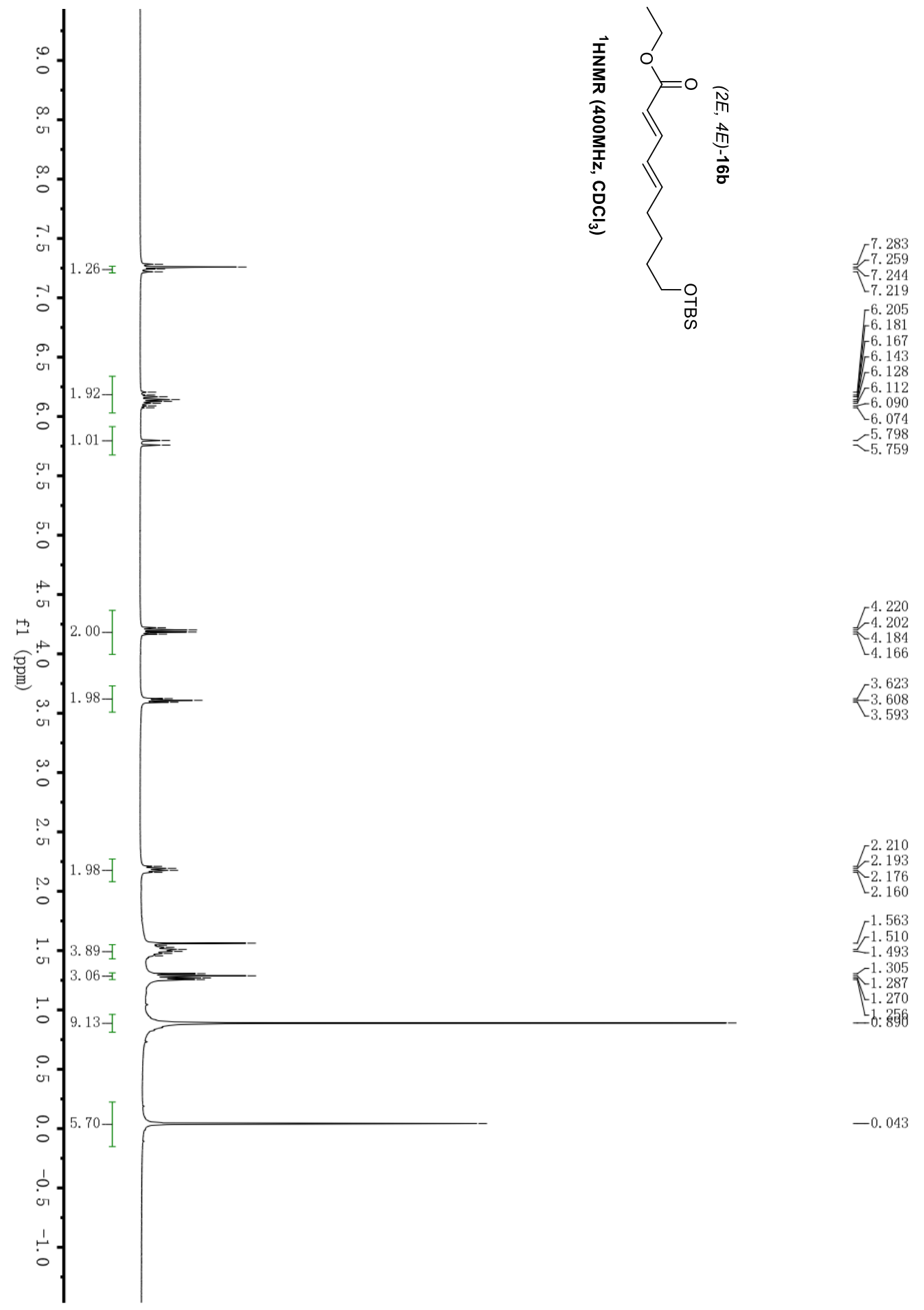

$-0.043$ 


$$
H
$$



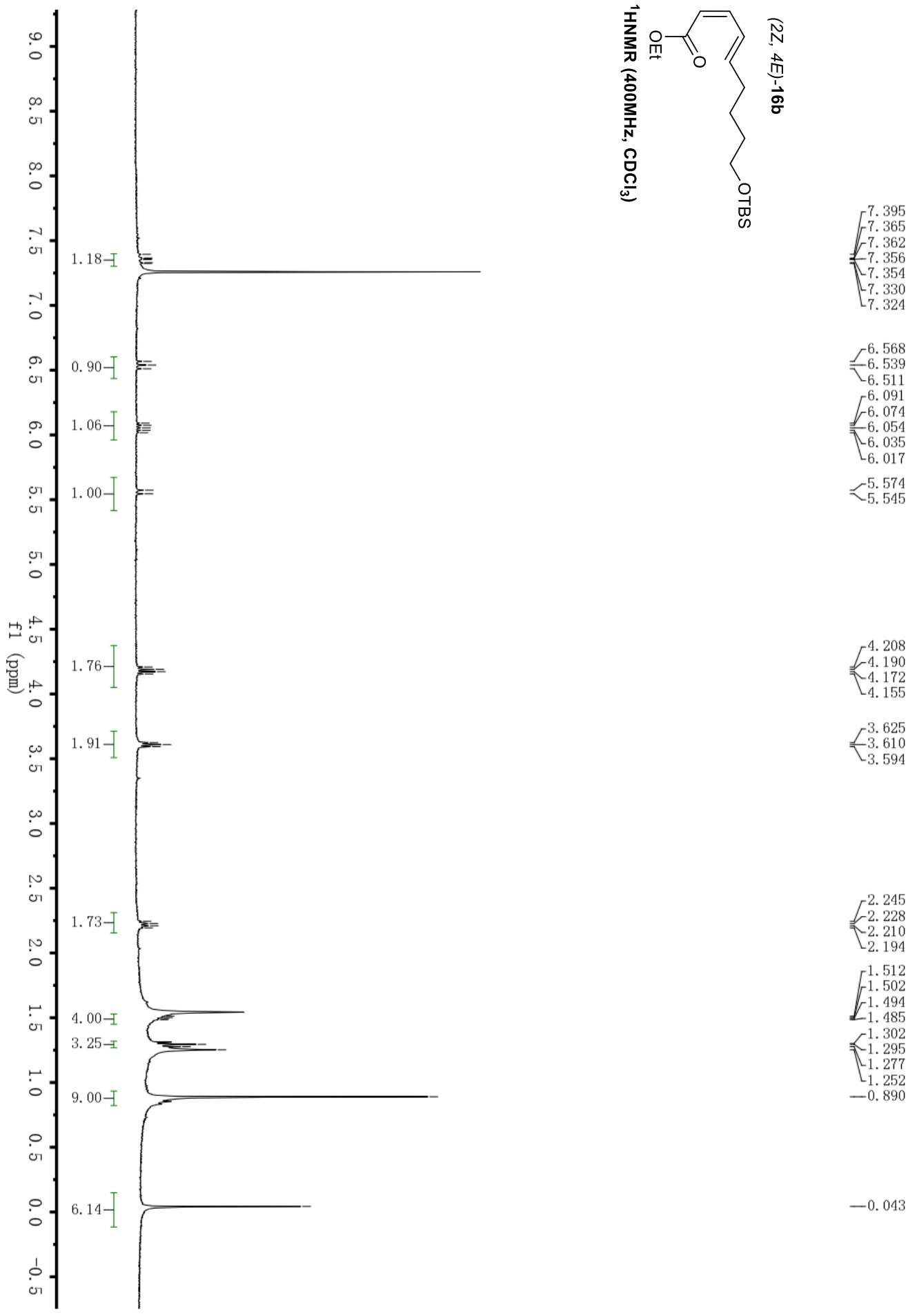

-6.511

6. 091

$-6.074$

-6. 035

-6. 017

$<_{5.545}^{5.574}$

4.208
-4.190
-4.172

-4.172
4.155

3.625
-3.610
3.594

2.245
-2.228
-2.210
2.194

$\Gamma^{1.512}$

1.502
-1.494
-1.485

-1. 302

$-1.295$

-1.277

$-0.890$

$-0.043$ 


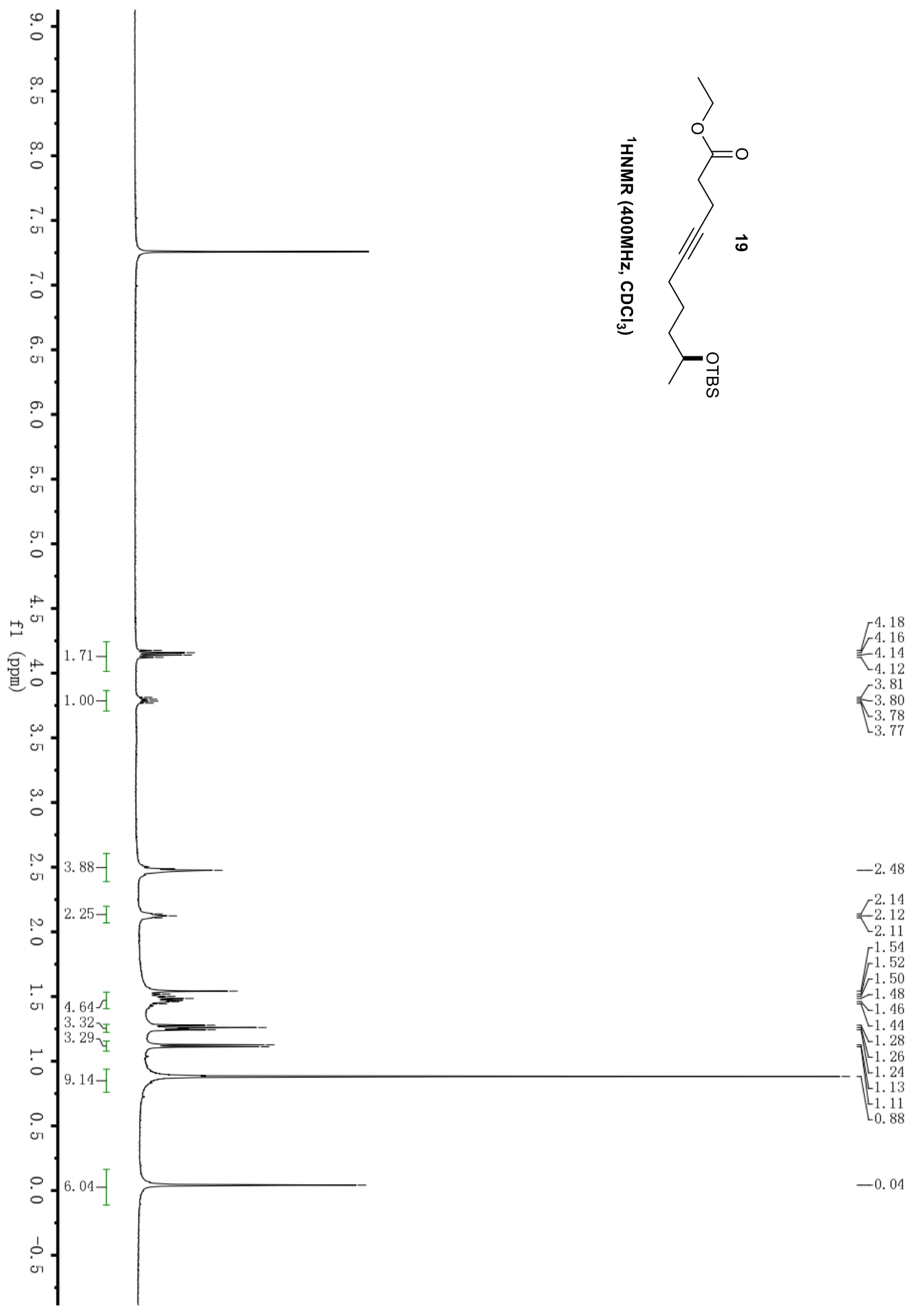




$$
15
$$




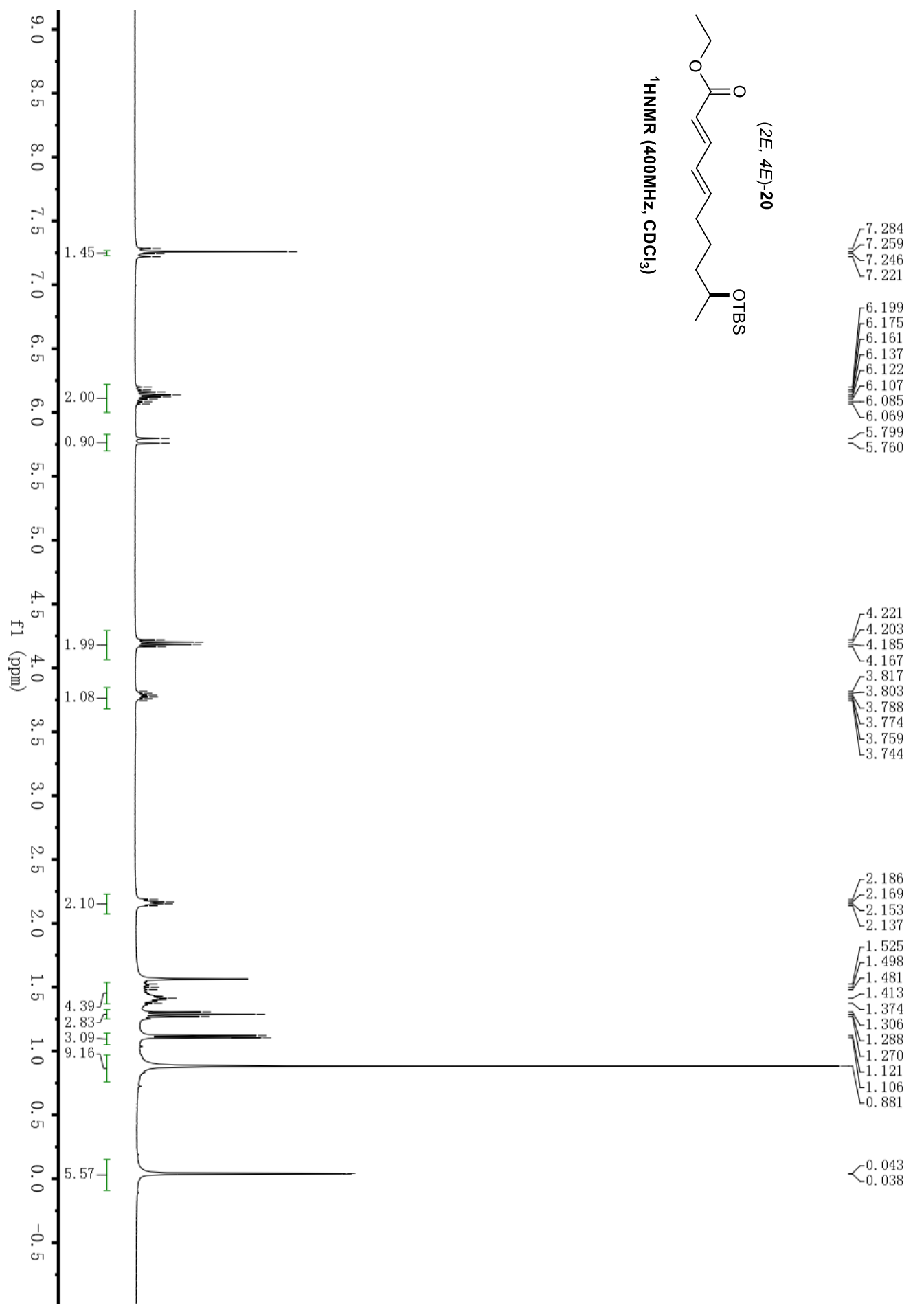

N 


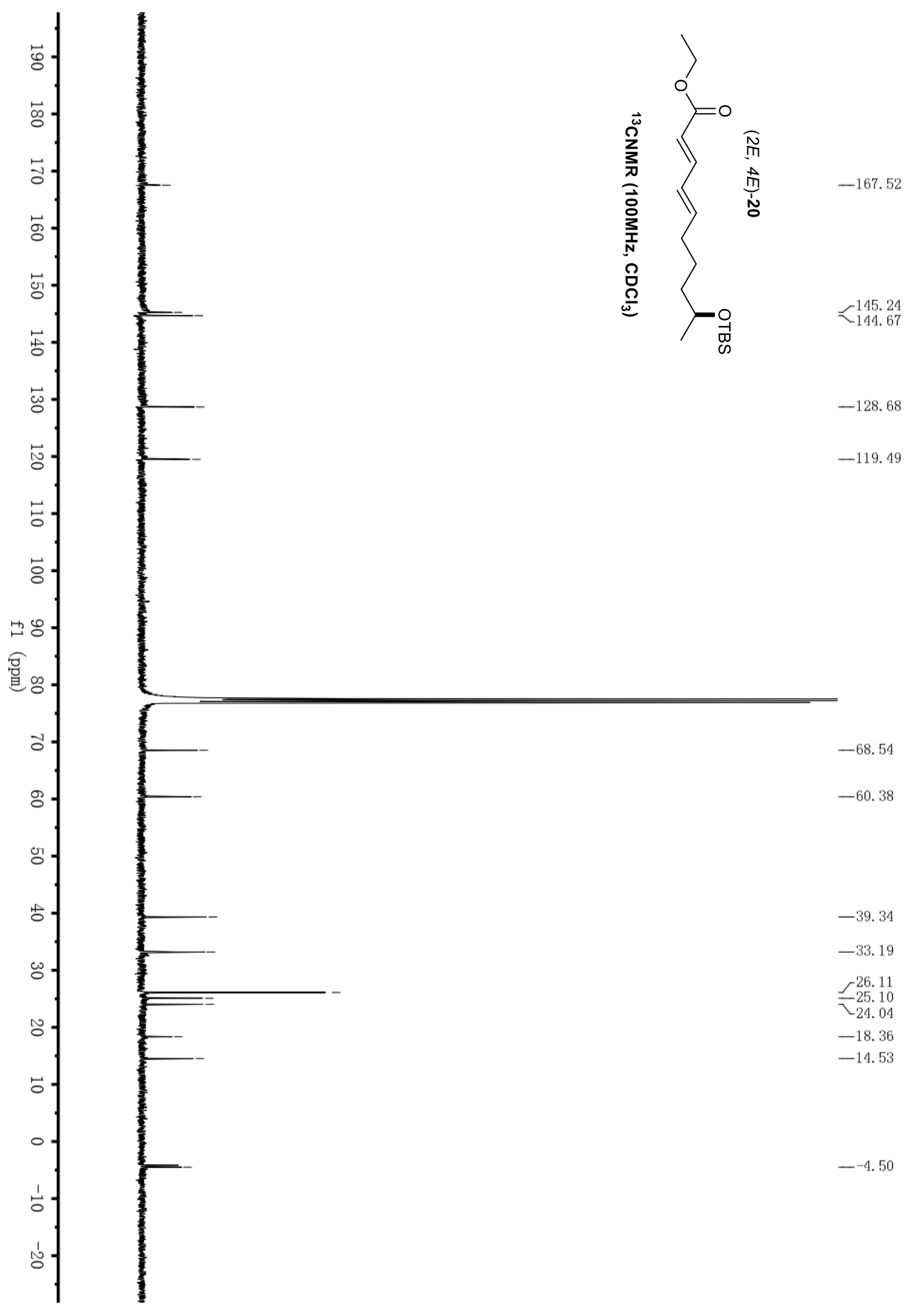



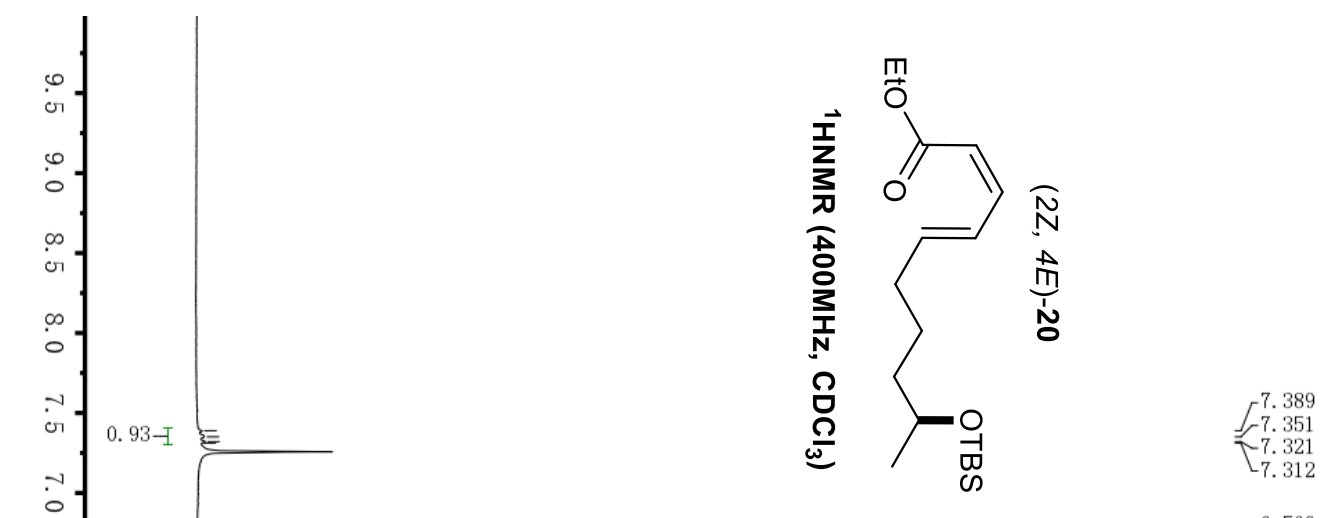

6.569
-6.559

-6.551
-6.512

$-6.063$

-6.044
6.026

6.098
-5.972

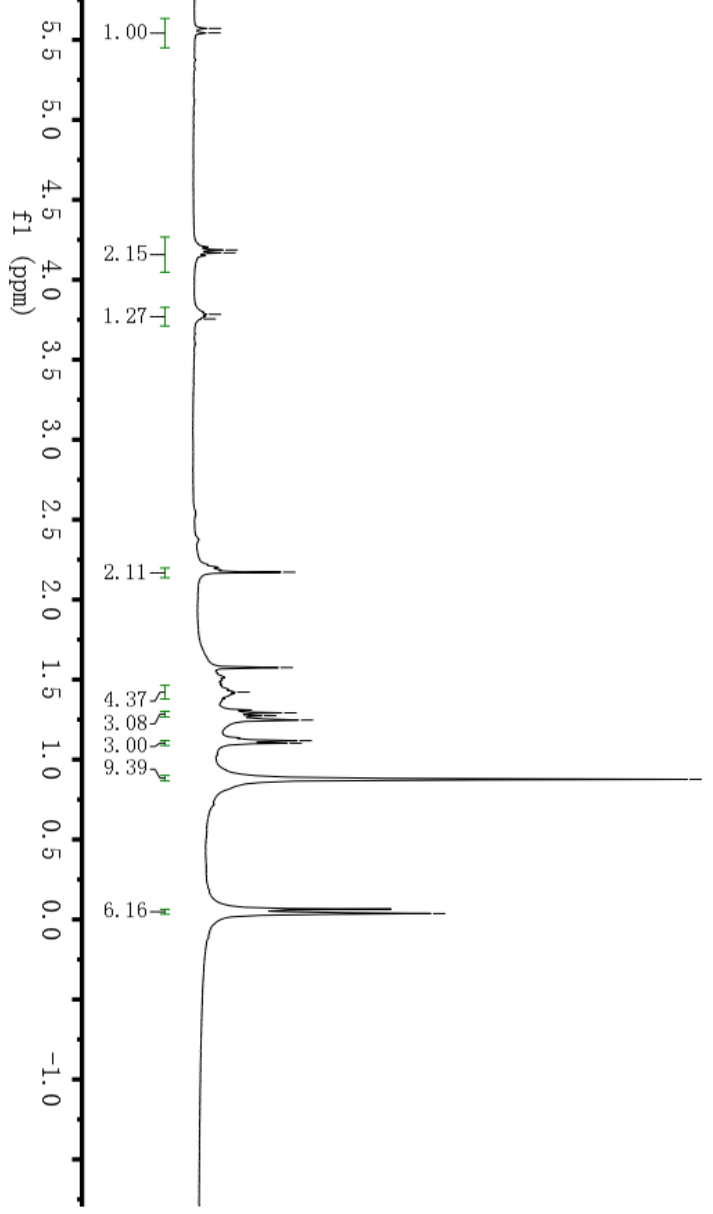

$-1.274$

-1. 118

-1.118
1.103

- .876

$-0.037$ 


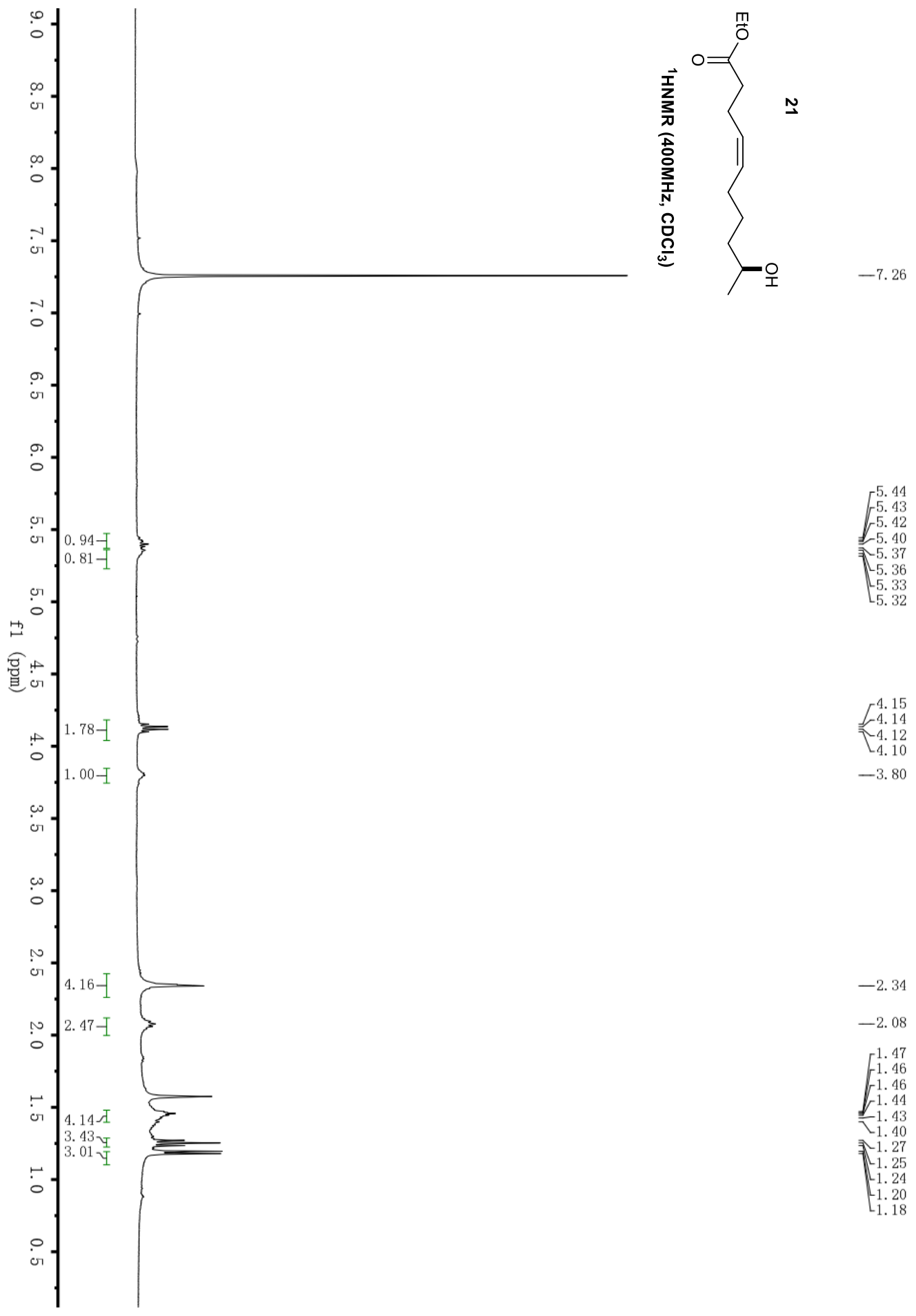




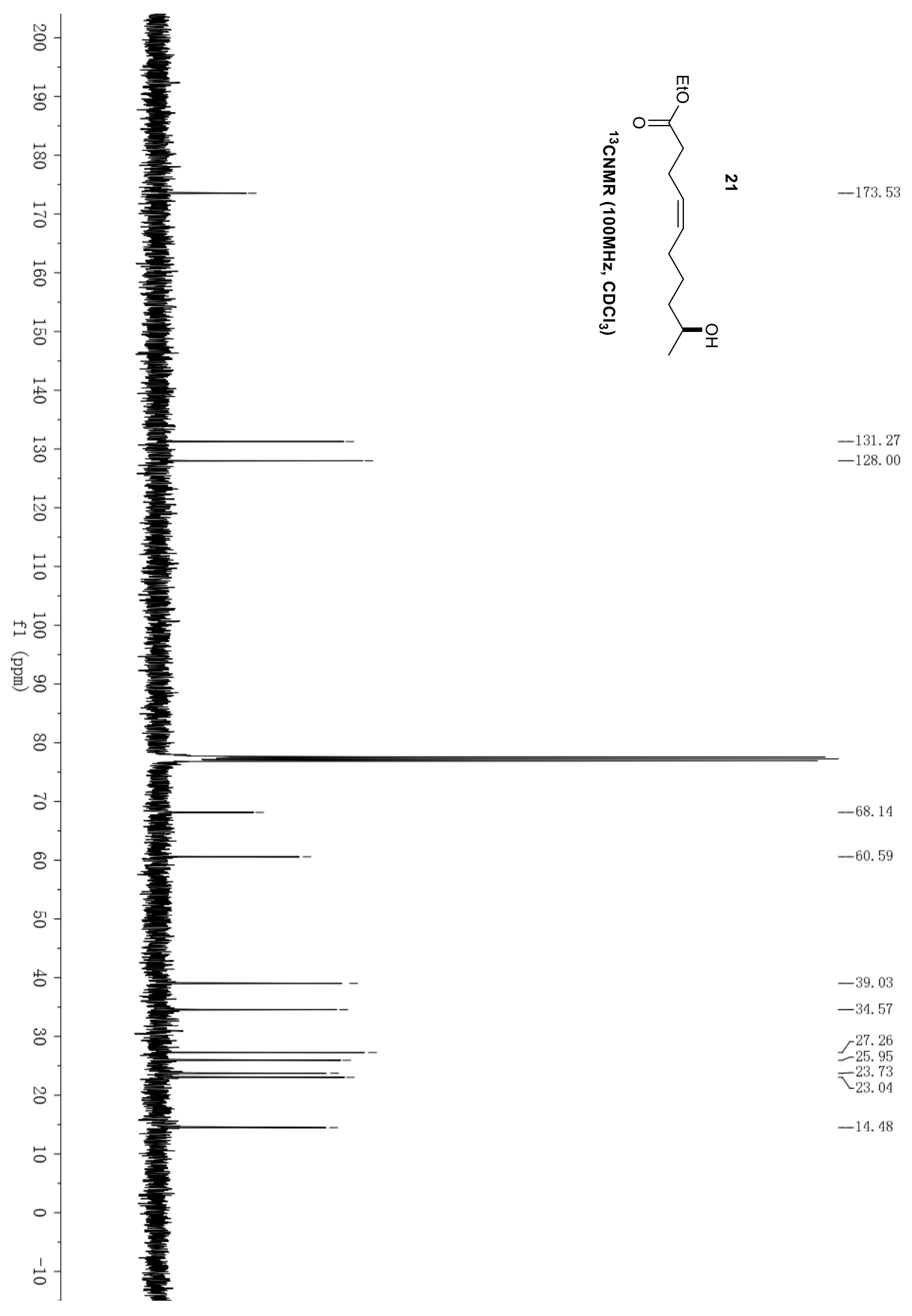



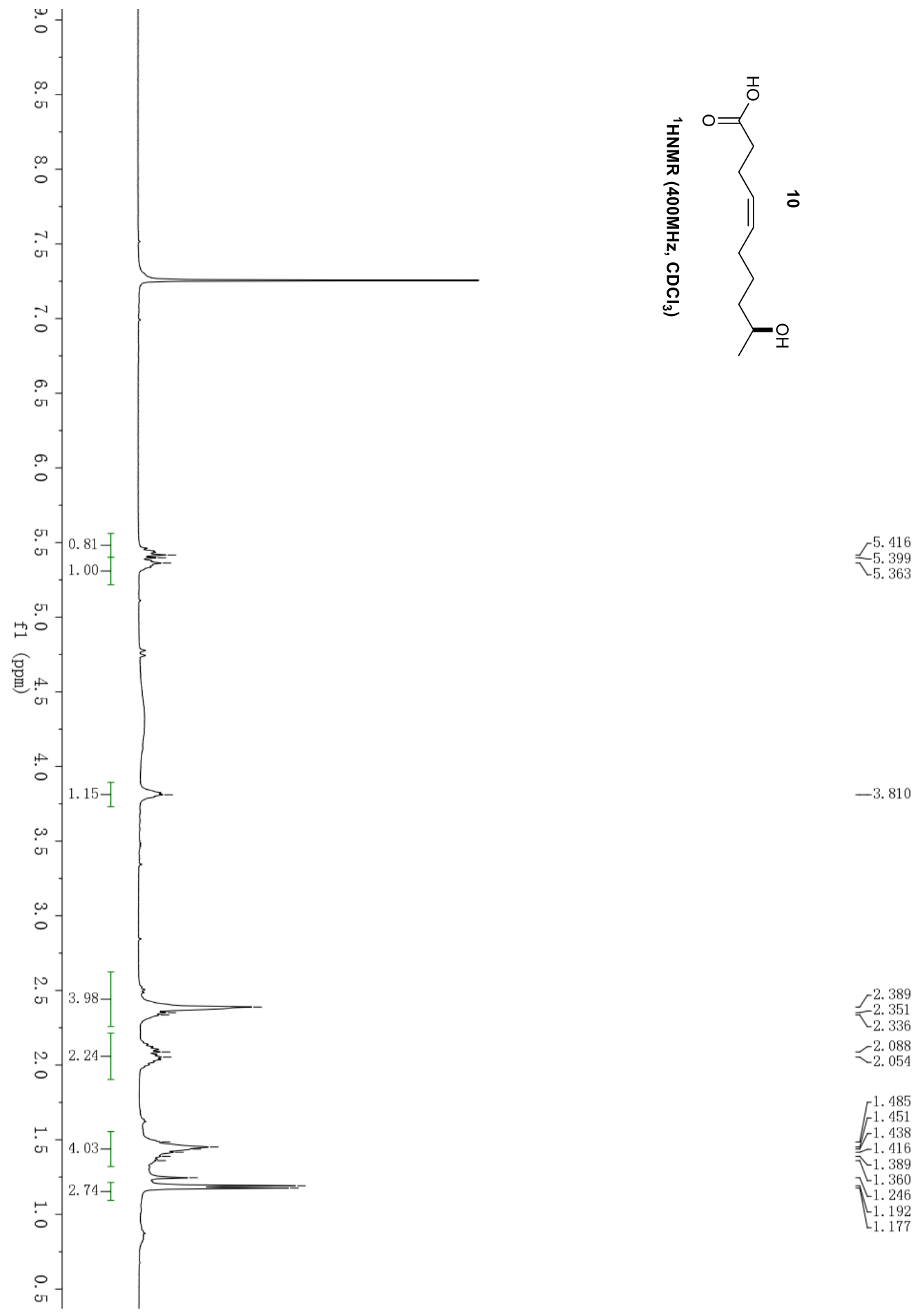

$-3.810$

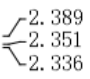

1.485
1.451

1.438
-1.416

-1.416
-1.389

-1. 389

ᄂ 1.246

- 1.246 


$$
k
$$




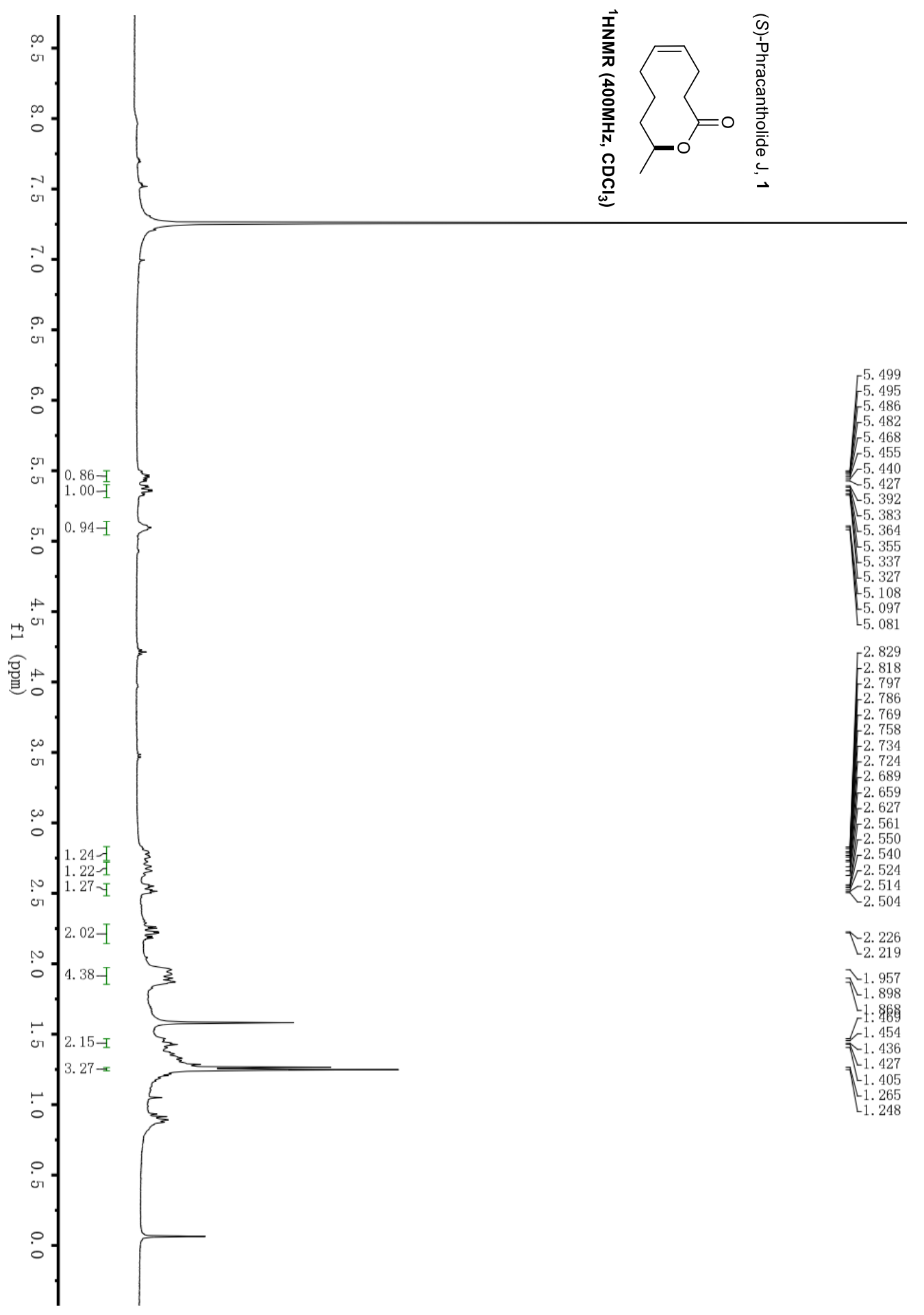




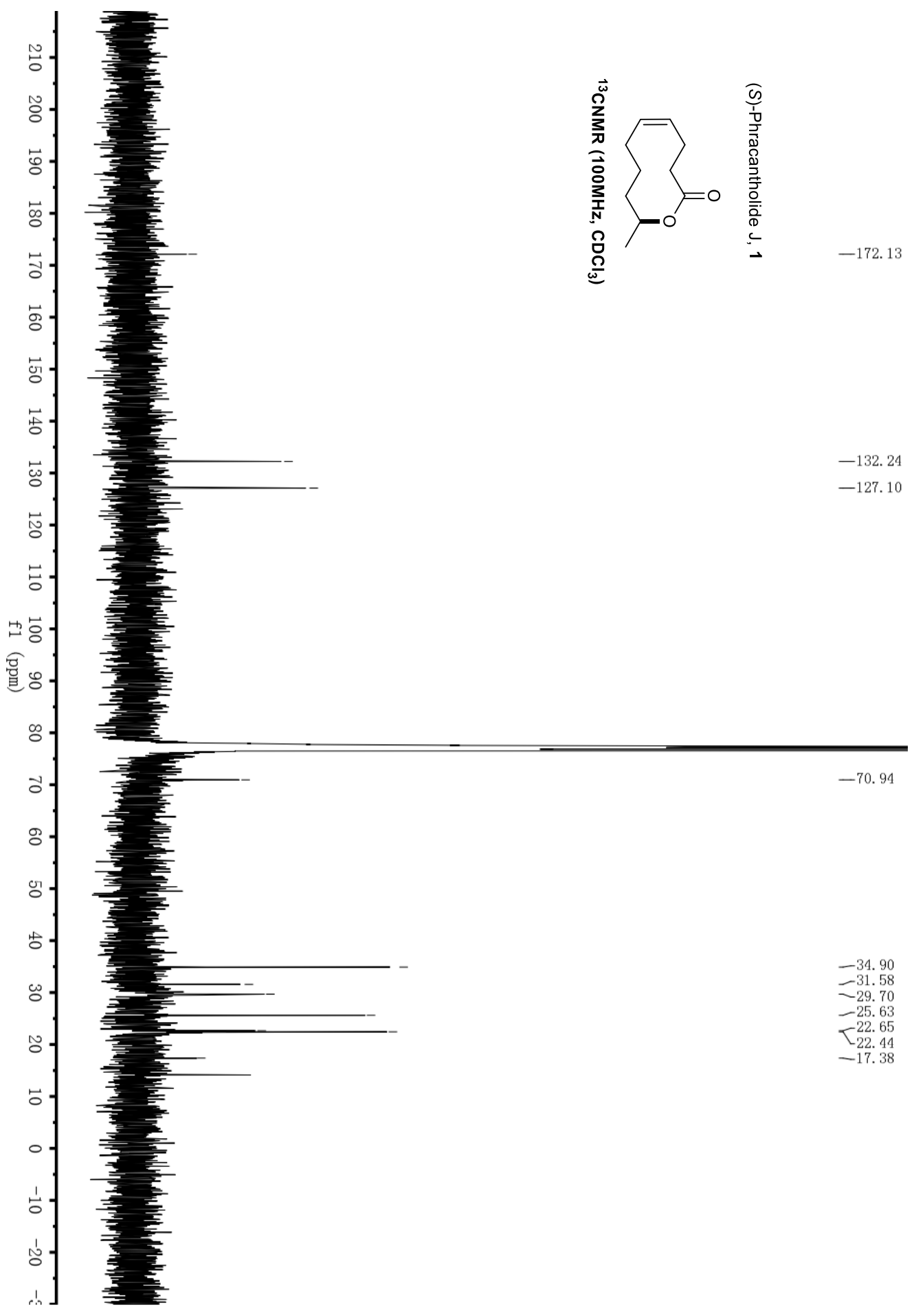

$\infty$ 